\title{
Kynurenic acid underlies sex-specific immune responses to COVID-19
}

\section{Authors}

Yuping Cai ${ }^{1}$, Daniel J. Kim ${ }^{2}$, Takehiro Takahashi ${ }^{2}$, David I. Broadhurst ${ }^{3}$, Shuangge Ma $^{4}$, Nicholas J.W. Rattray ${ }^{5}$, Arnau Casanovas-Massana ${ }^{6}$, Benjamin Israelow ${ }^{2,7}$, Jon Klein ${ }^{2}$, Carolina Lucas $^{2}$, Tianyang $\mathrm{Mao}^{2}$, Adam J. Moore ${ }^{6}$, M. Catherine Muenker ${ }^{6}$, Jieun $\mathrm{Oh}^{2}$, Julio Silva ${ }^{2}$, Patrick Wong ${ }^{2}$, Yale IMPACT Research team, Albert I. $\mathrm{Ko}^{6}$, Sajid A. Khan ${ }^{8}$, Akiko Iwasaki ${ }^{2,9}$, Caroline H. Johnson $^{1}$

${ }^{1}$ Department of Environmental Health Sciences, Yale School of Public Health, New Haven, CT 06510, USA

${ }^{2}$ Department of Immunobiology, Yale University School of Medicine, New Haven, CT 06520, USA

${ }^{3}$ Centre for Integrative Metabolomics \& Computational Biology, School of Science, Edith Cowan University, Joondalup, 6027, Australia

${ }^{4}$ Department of Biostatistics, Yale School of Public Health, New Haven, CT 06510, USA

${ }^{5}$ Strathclyde Institute of Pharmacy and Biomedical Sciences, University of Strathclyde, Glasgow G4 0RE, UK

${ }^{6}$ Department of Epidemiology of Microbial Diseases, Yale School of Public Health, New Haven, CT 06510, USA

${ }^{7}$ Department of Internal Medicine, Section of Infectious Diseases, Yale University School of Medicine, New Haven, CT 06520, USA

${ }^{8}$ Department of Surgery, Division of Surgical Oncology, Yale University School of Medicine, New Haven, CT 06520, USA

${ }^{9}$ Howard Hughes Medical Institute, Chevy Chase, MD 20815, USA 


\section{Abstract}

Coronavirus disease-2019 (COVID-19) has poorer clinical outcomes in males compared to females, and immune responses underlie these sex-related differences in disease trajectory. As immune responses are in part regulated by metabolites, we examined whether the serum metabolome has sex-specificity for immune responses in COVID-19. In males with COVID19, kynurenic acid (KA) and a high KA to kynurenine (K) ratio was positively correlated with age, inflammatory cytokines, and chemokines and was negatively correlated with $\mathbf{T}$ cell responses, revealing that KA production is linked to immune responses in males. Males that clinically deteriorated had a higher KA:K ratio than those that stabilized. In females with COVID-19, this ratio positively correlated with $\mathbf{T}$ cell responses and did not correlate with age or clinical severity. KA is known to inhibit glutamate release, and we observed that serum glutamate is lower in patients that deteriorate from COVID-19 compared to those that stabilize, and correlates with immune responses. Analysis of Genotype-Tissue Expression (GTEx) data revealed that expression of kynurenine aminotransferase, which regulates KA production, correlates most strongly with cytokine levels and aryl hydrocarbon receptor activation in older males. This study reveals that KA has a sex-specific link to immune responses and clinical outcomes, in COVID-19 infection.

\section{Main}

Sex-related differences in coronavirus disease-2019 (COVID-19) severity and morbidity exist, with the male sex being a risk factor ${ }^{1}$; male COVID-19 patients have an increased risk of admission (OR 1.68, 95\%CI=1.45-1.90) and in-hospital mortality (OR 1.87, 95\%CI=1.33-2.63) ${ }^{1}$. It was recently shown that hospitalized patients with moderate SARS-CoV-2 infection have elevated levels of certain inflammatory cytokines and chemokines, and sex-differences exist in these immune responses ${ }^{2}$. Furthermore, across all ages, female patients at baseline had a more robust $\mathrm{T}$ cell activation than males. Loss of $\mathrm{T}$ cell activation was correlated with older age in males, and this poorer $\mathrm{T}$ cell response was correlated with worse disease outcomes in males only ${ }^{2}$. Therefore, males and females have clear differences in COVID-19 immune responses that correlate with clinical course. 
Since immune responses are regulated, in part, by metabolites, it is possible that sex-related differences in metabolism could affect the host immune response to SARS-CoV-2 infection. For instance, specific metabolites are required for macrophage, neutrophil, and $\mathrm{T}$ cell functions, enhancing glycolytic and fatty acid synthesis pathways in these cells ${ }^{3}$. Conversely, immune stimulation can also elicit metabolic reprograming in cells, thereby affecting disease trajectory by altering metabolite abundance ${ }^{4}$. In addition to the metabolic requirements of the host immune system, viruses also require host-derived metabolites and lipids ${ }^{5}$. Thus, utilization of metabolic substrates for viral replication could affect metabolite availability required for immune responses.

\section{Metabolites correlate with COVID-19}

To address how metabolites might mediate the sex-related differences in COVID-19 immune response, we first used an untargeted metabolomics workflow with multivariable logistic regression to identify serum metabolites associated with COVID-19. Serum samples were collected from COVID-19 patients ( $n=22$ females and $n=17$ males) on the day of enrollment into the IMPACT study at Yale New Haven Hospital (CT, USA). Samples were taken from patients 37 days after hospital admission after confirmation of COVID-19 infection who (1) were not immediately triaged to the intensive care unit, (2) had not received tocilizumab, and (3) had not received high dose corticosteroids (Cohort A described in Takahashi et al. ${ }^{2}$ ). Uninfected healthcare worker (HCW) controls ( $n=10$ females and $n=10$ males) were included in the analysis. There was a statistically significant difference in age between the COVID-19 patients and HCWs, which was adjusted for in our models (Extended Data Table 1). We first carried out metabolite identification on detected signals that were present in the serum metabolome of quality control samples pooled from both COVID-19 patients and HCWs. We positively identified 75 metabolites with the highest confidence (Extended Data Table 2). Regression analysis revealed that 17 metabolites were associated with COVID-19 status after adjustment for age, BMI, sex, and multiple comparisons (Extended Data Table 3). Glutamate, cysteine-S-sulfate, palmitoleic acid, arachidonic acid, lysophosphatidylethanolamine (LPE) (22:6), uracil and myristic acid were positively associated with COVID-19. Whereas glutamine, 3-methylxanthine, tryptophan, proline, citrulline, homoserine, 2,3-dihydroxybenzoic acid, lysophosphatidic acid (LPA) (18:2), LPA (20:2), and lysophosphatidylcholine (14:0) were negatively associated with COVID-19.

\section{Metabolites correlate with immune response in a sex-specific manner}


Next, we examined how the 75 positively identified serum metabolites from both COVID-19 patients and HCWs might correlate with immune markers (cytokines and chemokines levels in plasma, T cells, B cells, NK-T cells, NK cells, monocytes, macrophages, and dendritic cells in peripheral blood mononuclear cells (PMBCs)) that were previously measured from the same individuals $^{2}$ (Fig. 1). In COVID-19 patients, we observed 36 correlations between immune markers and metabolites with $|\mathrm{R}|>0.5$ and $p$ value $<0.05$ (Extended Data Fig. 1, Supplementary Table 1). However, after stratification by sex, additional correlations were observed between metabolites and immune markers for each sex independently, suggesting that sex-specific immune responses could be linked to metabolism (Extended Data Fig. 1, Supplementary Table 2).

Further examination revealed that kynurenic acid (KA), an endogenous ligand of the aryl hydrocarbon receptor (AhR) that regulates immunes responses ${ }^{6}$, had positive correlations with immune markers (Fig. 1). Many of these positive correlations were observed in male patients including IL1RA, IL6, IL10, TNFa, M-CSF, SCF, CX3CL1, CXCL9, CXCL13, CCL1, CCL21, and CCL22. In addition, KA in males was negatively associated with T cell number, naïve CD8 T cells, CD4 effector memory (CD4Tem), and CD8 effector memory (CD8Tem) T cells (Figs. 2a, 2b). In female patients, KA was positively associated only with IL12p40, CCL3, CXCL9, and SCF (Fig. 2b). In summary, sex-specific differences in correlations between metabolites and immune responses were observed in COVID-19 patients, wherein KA had the most prominent connection to immune response in males.

\section{Kynurenic acid is associated with a sex-specific immune response}

To further understand the sex-specific correlation of KA to immune markers, we examined the relationship between KA and kynurenine (Fig. 2b). Kynurenine (K) is a product of tryptophan metabolism that is converted to KA by kynurenine aminotransferases (KATs), which are encoded by KYAT genes (Fig. 2c). Of note, tryptophan was inversely associated with COVID-19 disease, as noted in Extended Data Table 3. We examined the ratio of KA:K in patients with COVID-19 as a surrogate for KAT-mediated production of KA from $\mathrm{K}^{7}$. In males, we observed that a high KA:K was positively correlated with IL6, CCL1, CCL21, TNF $\alpha$, M-CSF, NK cells, and CD8

terminally differentiated effector memory (Temra) T cells (Fig. 2b). A high KA:K was negatively correlated with sCD40L, PDGFAA, PDGFAB/BB, monocytes, PD1+TIM3+CD8 T cells, CD38+HLA-DR+CD8+ T cells, naïve T cells, and IL6 ${ }^{+} \mathrm{CD} 4 \mathrm{~T}$ cells (Fig. 2c). Of note, a high 
$\mathrm{KA}: \mathrm{K}$ was positively correlated with $\mathrm{T}$ cell activation in females, but overall $\mathrm{T}$ cell numbers showed a negative correlation with the ratio of KA:K in males with COVID-19 (Fig. 2c).

We also observed that KA:K and KA positively correlated with age in males with COVID-19 (Fig. 2d, Extended Data Fig. 2a). KA had a low positive correlation to age in females with COVID19, but the ratio of KA:K was not correlated (Extended Data Fig. 2a, Fig. 2d). In HCWs, KA negatively correlated with age only in males (Extended Data Fig. 2a), while KA:K negatively correlated with age in both males and females (Fig. 2d).

Closer examination of other metabolites involved in $\mathrm{K}$ and $\mathrm{KA}$ metabolism revealed additional correlates of the cellular immune response during COVID-19. The microbial catabolite of tryptophan, indole-3-lactic acid ${ }^{8}$, was positively associated with IL4+CD4 and CD38+HLADR+CD8 cells in males (Extended Data Fig. 2b). In females, indole-3-lactic acid was negatively associated with plasma levels of G-CSF, M-CSF, and CXCL10; K was positively associated with IL2, CCL15, CXCL13, and SCF; and tryptophan was positively correlated with follicular CD8 T cells (Fig. 2b).

To evaluate whether the sex-specific association between KA and the immune response, which was observed in males with COVID-19, is a phenomenon also present in healthy individuals, we analyzed tissue-specific expression data from the Genotype-Tissue Expression (GTEx) Project ${ }^{9}$. While KYAT genes generally tended to have more positive correlations with cytokines in males compared to females, KYAT3 had particularly stronger correlations in a subset of tissues (including the brain and colon), many of which are classically involved in COVID-19 (Extended Data Fig. 3). Within the brain, we found that these positive correlations with cytokines were specific to older males (aged > 60 years old) (Extended Data Fig. 4a). Because KA is a ligand for AhR which regulates immune responses and inflammation ${ }^{6}$, we analyzed $\mathrm{AhR}$ activation using a previously defined score ${ }^{10}$ and found that AhR activation correlates most positively with KYAT3 expression in older males in brain and muscle, while closely correlating in colon (Extended Data Fig. 4b). Correlations in the brain became even more pronounced when analyzing only the AhR target gene CYP1B1, which is classically used to follow AhR activation in the brain (Extended Data Fig. 4c) ${ }^{11}$. We also show that KYAT3 expression decreases with age in both males and females, which is consistent with the decreasing ratios of $\mathrm{KA}: \mathrm{K}$ observed in $\mathrm{HCW}$ control samples (Fig. 2e). In summary, these data suggest that older males are uniquely sensitive to increases in KA levels, 
reacting disproportionately with increased levels of inflammatory cytokines, likely as part of a broader AhR activation.

\section{Kynurenic acid correlates with disease severity in a sex-specific manner}

As KA levels correlate with numerous immune markers, and these immune markers have been previously linked disease progression ${ }^{2}$, we examined whether KA was directly associated with disease severity. We used previously defined clinical scores to classify disease severity in COVID19 patients as deteriorated (males $n=6$, females $n=6$ ) or stabilized (males $n=11$, females $n=16)^{2}$. KA levels were not significantly different between deteriorated and stabilized patients, or after additional stratification by sex. However, KA was positively correlated with CXCL9, IL6, IL12p40, CCL1, CCL3, CCL15, CCL21, CCL27, SCF, M-CSF, and G-CSF in patients that deteriorated with COVID-19 $(|\mathrm{R}|>0.5$ and $p$ value<0.05). In stabilized patients, KA was also positively correlated with CXCL9 and CX3CL1 (Figs. 3a, b). We further examined whether KA:K was correlated with disease severity by sex. Males who deteriorated had a significantly higher $\mathrm{KA}: \mathrm{K}$ compared to those that stabilized, whereas there was no difference in $\mathrm{KA}: \mathrm{K}$ between females based on clinical course (Fig. 3c). Furthermore, a high KA:K was positively correlated with CXCL9 and CCL1 in males that deteriorated, but this correlation was not seen in patients that stabilized, or in females (Fig. 3d).

We also examined whether any of the 17 metabolites associated with COVID-19 status (Extended Data Table 3) were correlated with disease severity. We observed that only glutamate was associated with disease trajectory, and a significantly higher glutamate level was observed in stabilized patients compared to those that deteriorated (Fig. 4a); incidentally, KA is a glutamate receptor antagonist, thus high KA production could inhibit glutamate release ${ }^{12}$. Correlation analysis revealed that Eotaxin2, IL5, CD4 T cells, CD4rnTreg negatively correlated with glutamate in deteriorated patients, whereas IL6, CD8 T cells and GzB+CD8 T cells positively correlate (Fig. 4b). Further stratification by sex, showed a similar trend where stabilized patients had higher levels of glutamate than deteriorated patients within each sex group (Fig. 4a). Correlation analysis of immune markers with glutamate by sex revealed a negative correlation to Eotaxin 2 and positive correlation to IL6, only in females that deteriorate. CD4 T cells negatively correlate and GzB+CD8 T cells positively correlate with glutamate, only in males that deteriorate. IL5 negatively correlates with glutamate in males who deteriorate whereas it negatively correlates with glutamate in females 
that stabilize. CD8 $\mathrm{T}$ cells positively correlate with glutamate in males that deteriorate and negatively correlate in males that stabilize (Fig. 4c). These data suggest that low levels of glutamate may contribute to poorer disease progression in patients with COVID-19 by regulating immune responses. In addition, a high $\mathrm{KA}: \mathrm{K}$ is correlated to poorer prognosis only in male COVID-19 patients.

\section{Discussion}

Patients with severe COVID-19 disease experience a "cytokine storm", characterized by the elevation of pro-inflammatory cytokines and aggressive inflammatory response ${ }^{13}$, and sexspecificity in immune response has been previously reported that could underlie differences in clinical outcomes ${ }^{2}$. Our analysis of serum metabolites from COVID-19 patients, reveals that KA and the ratio of $\mathrm{KA}: \mathrm{K}$ has a strong relationship to sex-specific immune response and clinical disease course. Importantly, a previous study revealed that serum metabolites in tryptophan and $\mathrm{K}$ metabolism correlate with IL6 in a sex-aggregated cohort of COVID-19 patients, but sexspecificity was not examined ${ }^{14}$. Our study shows that, in males, a high ratio of KA:K was positively correlated with increased levels of cytokines/chemokines and portends clinical deterioration. On the other hand, a negative association was observed with Eotaxin, sCD40L, PDGFs, and T cells, indicating that males with a high KA:K may have a poorer response to inflammation associated with COVID-19, including decreased eosinophil recruitment and T cell activation ${ }^{15,16}$. A previous study showed that PDGFs associate with better prognosis from COVID-19 if the patients have lower cytokine levels, supporting the association between a higher $\mathrm{KA}: \mathrm{K}$ and poorer outcomes in males $^{17}$. In females with COVID-19, a high KA:K positively correlated with a small number of cytokines and also $\mathrm{T}$ cell activation, but in contradistinction to males, high $\mathrm{KA}: \mathrm{K}$ was not associated with disease severity. These results therefore support the role of K metabolism in sexrelated differences previously reported in immune responses to COVID- $19^{2}$.

Analyzing gene expression data from GTEx, we found that older males (but not females or younger males) appear to have exquisite sensitivity to changes in KYAT gene expression (which we used as a proxy for KA levels), whereby natural increases in KYAT expression are met with concomitant natural increases in tissue cytokine expression. It is worthwhile to note that the tissues exhibiting these sex-specific correlations - including brain, muscle, kidney, and colon - are those that are 
commonly implicated in symptoms of COVID-19 patients such as anosmia, myalgia, acute kidney injury, and gastrointestinal distress.

Given its role in regulating the immune system and inducing pro-inflammatory cytokines like IL $6^{6}$, modulations in the AhR signaling pathway likely account for this differential response among older males. In support of this hypothesis, we show that AhR activation is most strongly associated with KYAT3 expression in healthy older males. Also, studies have already shown that male rodents have a more toxic response to stereotypical AhR agonists like TCDD ${ }^{18}$. Furthermore, testosteronemediated signaling is known to inhibit AhR activity ${ }^{19}$, and given the decreasing serum levels of testosterone seen in older males ${ }^{20}$, it seems plausible that healthy older males could be naturally susceptible to greater AhR activation by endogenous ligands.

In the context of COVID-19 infection, patients presented with elevated KAT activity (as suggested by high KA:K ratios), especially among deteriorating male patients. A recent study has demonstrated that a similar AhR induction occurs in the context of murine coronavirus infection, inducing IDO-2 expression ${ }^{17}$. Notably, two major risk factors for COVID-19, type 2 diabetes and obesity, have already been shown to have increased AhR ligand activity ${ }^{21,22}$. Such an influx of endogenous AhR ligands, combined with an already elevated susceptibility for AhR activation, therefore, would pose a significantly elevated risk of developing a cytokine storm, specifically in older male patients.

The analysis in this study also revealed discrete serum metabolites associated with COVID-19 that may account for some of the varying clinical outcomes in these patients. For instance, metabolites that were positively associated with COVID-19 (Extended Data Table 3) have inflammatory (palmitoleic ${ }^{23}$ and arachidonic acids ${ }^{24}$ ) and neurological (glutamate ${ }^{25}$ and cysteine-S-sulfate ${ }^{26}$ ) roles. Metabolites negatively associated with COVID-19, are involved in the urea cycle and nitric oxide (NO) synthesis pathway (proline, citrulline, and glutamine ${ }^{27}$ ). The NO synthesis pathway mediate responses to pro-inflammatory cytokines, macrophages, and neutrophils. Low levels of citrulline have been observed in patients with acute respiratory distress syndrome ${ }^{28}$ and can cause NO synthase uncoupling and decreased NO synthesis, which is important for vascular function and endothelial cell function ${ }^{29}$. It was recently suggested that therapeutic NO could be used to improve pulmonary vascular function in COVID-19 ${ }^{30}$. Of the 17 metabolites associated with COVID-19 status, only glutamate was associated with disease severity. In addition, sex-specific 
correlations between immune responses and glutamate were observed by disease severity. Males that deteriorated from COVID-19 had positive correlations between glutamate and CD8 T cells, and a negative correlation with CD4 T cells. A previous study showed that higher innate immune cytokine levels are associated with clinical deterioration in females with COVID- $19^{2}$, here we observed that IL6 was positively correlated with glutamate only in females that deteriorate. Increasing levels of glutamate have also been shown to decrease IL5 secretion ${ }^{31}$, and we observed a negative correlation between glutamate and IL5 in males that deteriorate and also in females that stabilize, furthermore, Eotaxin2 negatively correlates with glutamate in females that deteriorate. Similar to Eotaxin2, IL5 is also linked to eosinophil activation, therefore in females, glutamate may be important in regulating eosinophilia in COVID-19. Incidentally, KA is a glutamate receptor antagonist. Glutamate receptors are expressed on the surface of T cells and expression of these receptors is triggered by $\mathrm{T}$ cell activation ${ }^{32}$. Glutamate transporters have also been described in various immune cells ${ }^{32}$, therefore, this correlation could be therefore reflective of the actions of KA on glutamate levels and also immune cell responses to COVID-19 ${ }^{33}$.

Because our study did not analyze non-COVID individuals exhibiting similar clinical symptoms to COVID-19 patients, it remains a possibility that elevated KA and KA:K may lead to elevated cytokines and more broadly mediate the inflammatory symptoms of other pathologies. This possibility, however, does not detract from our observations in COVID-19 patients or the potential of AhR as a therapeutic target in COVID-19.

In summary, we have identified serum metabolites associated with COVID-19 clinical course, immune response and sex-specific differences. Among these metabolites, perhaps the most salient discovery is the identification of KA as a metabolite associated with sex, age, increased disease severity, and elevated cytokine and chemokine levels. KA is a ligand for AhR, and when activated, $\mathrm{AhR}$ is a master regulator of immune responses and inflammation. Sex-specific agonism of AhR has yet to be reported in humans, but appears to be a prominent feature in COVID-19 disease, potentially underlying the cytokine storm and dampening of T cell activation. In addition, KA is known to dampen glutamate release ${ }^{12}$, and we observed lower levels of glutamate in patients that deteriorate compared to those that stabilize. Further investigation into the relevance of KA, KAT, and AhR activation in COVID-19 and the role of glutamate in clinical outcomes will be of utmost importance, particularly for understanding the sex-specific differences in immune response and patient outcomes. As we learn more about the impact of the metabolome on COVID-19 disease 
medRxiv preprint doi: https://doi.org/10.1101/2020.09.06.20189159; this version posted September 8, 2020. The copyright holder for this preprint (which was not certified by peer review) is the author/funder, who has granted medRxiv a license to display the preprint in perpetuity.

It is made available under a CC-BY-NC-ND 4.0 International license .

course, clinicians may find that modulating metabolite levels, either through enteral nutrition or targeted metabolic enzymes may alter disease trajectory.

\section{REFERENCES}

1 McPadden, J. et al. Clinical Characteristics and Outcomes for 7,995 Patients with SARSCoV-2 Infection. medRxiv, doi:10.1101/2020.07.19.20157305 (2020).

2 Takahashi, T. et al. Sex differences in immune responses that underlie COVID-19 disease outcomes. Nature, doi:10.1038/s41586-020-2700-3 (2020).

3 Ganeshan, K. \& Chawla, A. Metabolic regulation of immune responses. Annu Rev Immunol 32, 609-634, doi:10.1146/annurev-immunol-032713-120236 (2014).

4 O'Neill, L. A., Kishton, R. J. \& Rathmell, J. A guide to immunometabolism for immunologists. Nature reviews. Immunology 16, 553-565, doi:10.1038/nri.2016.70 (2016).

5 Song, J. W. et al. Omics-Driven Systems Interrogation of Metabolic Dysregulation in COVID-19 Pathogenesis. Cell metabolism, doi:10.1016/j.cmet.2020.06.016 (2020).

6 Wirthgen, E., Hoeflich, A., Rebl, A. \& Günther, J. Kynurenic Acid: The Janus-Faced Role of an Immunomodulatory Tryptophan Metabolite and Its Link to Pathological Conditions. Frontiers in Immunology 8, doi:10.3389/fimmu.2017.01957 (2018).

7 Liu, H. et al. The Metabolic Factor Kynurenic Acid of Kynurenine Pathway Predicts Major Depressive Disorder. Frontiers in psychiatry 9, 552-552, doi:10.3389/fpsyt.2018.00552 (2018).

8 Gao, J. et al. Impact of the Gut Microbiota on Intestinal Immunity Mediated by Tryptophan Metabolism. Front Cell Infect Microbiol 8, 13-13, doi:10.3389/fcimb.2018.00013 (2018).

9 Carithers, L. J. et al. A Novel Approach to High-Quality Postmortem Tissue Procurement: The GTEx Project. Biopreservation and biobanking 13, 311-319, doi:10.1089/bio.2015.0032 (2015).

10 Kim, D. J. et al. Vitamin B12 and folic acid alleviate symptoms of nutritional deficiency by antagonizing aryl hydrocarbon receptor. Proceedings of the National Academy of Sciences 117, 15837-15845, doi:10.1073/pnas.2006949117 (2020).

11 Jacob, A. et al. Aryl hydrocarbon receptor-dependent upregulation of Cyp1b1 by TCDD and diesel exhaust particles in rat brain microvessels. Fluids Barriers CNS 8, 23-23, doi:10.1186/2045-8118-8-23 (2011). 
12 Réus, G. Z. et al. Kynurenine pathway dysfunction in the pathophysiology and treatment of depression: Evidences from animal and human studies. J Psychiatr Res 68, 316-328, doi:10.1016/j.jpsychires.2015.05.007 (2015).

13 Ragab, D., Salah Eldin, H., Taeimah, M., Khattab, R. \& Salem, R. The COVID-19 Cytokine Storm; What We Know So Far. Frontiers in Immunology 11, doi:10.3389/fimmu.2020.01446 (2020).

14 Thomas, T. et al. COVID-19 infection alters kynurenine and fatty acid metabolism, correlating with IL-6 levels and renal status. JCI Insight 5, doi:10.1172/jci.insight.140327 (2020).

15 Paige, L. in Cytokine Effector Functions in Tissues Vol. 1 Ch. 12, (Academic Press, 2017).

16 Chung, H. W. \& Lim, J.-B. Clinical significance of elevated serum soluble CD40 ligand levels as a diagnostic and prognostic tumor marker for pancreatic ductal adenocarcinoma. Journal of translational medicine 12, 102, doi:10.1186/1479-5876-12-102 (2014).

17 Lucas, C. et al. Longitudinal analyses reveal immunological misfiring in severe COVID19. Nature, doi:10.1038/s41586-020-2588-y (2020).

18 Enan, E., Overstreet, J. W., Matsumura, F., VandeVoort, C. A. \& Lasley, B. L. Gender differences in the mechanism of dioxin toxicity in rodents and in nonhuman primates. Reprod Toxicol 10, 401-411, doi:10.1016/0890-6238(96)83995-5 (1996).

19 Jana, N. R. et al. Cross-talk between 2,3,7,8-tetrachlorodibenzo-p-dioxin and testosterone signal transduction pathways in LNCaP prostate cancer cells. Biochemical and biophysical research communications 256, 462-468, doi:10.1006/bbrc.1999.0367 (1999).

20 Harman, S. M., Metter, E. J., Tobin, J. D., Pearson, J. \& Blackman, M. R. Longitudinal effects of aging on serum total and free testosterone levels in healthy men. Baltimore Longitudinal Study of Aging. The Journal of clinical endocrinology and metabolism 86, 724-731, doi:10.1210/jcem.86.2.7219 (2001).

21 Roh, E. et al. Serum aryl hydrocarbon receptor ligand activity is associated with insulin resistance and resulting type 2 diabetes. Acta diabetologica 52, 489-495, doi:10.1007/s00592-014-0674-z (2015).

22 Kerley-Hamilton, J. S. et al. Obesity is mediated by differential aryl hydrocarbon receptor signaling in mice fed a Western diet. Environmental health perspectives 120 , 1252-1259, doi:10.1289/ehp.1205003 (2012).

23 de Souza, C. O. et al. Palmitoleic Acid has Stronger Anti-Inflammatory Potential in Human Endothelial Cells Compared to Oleic and Palmitic Acids. Mol Nutr Food Res 62, e1800322, doi:10.1002/mnfr.201800322 (2018). 
24 Samuelsson, B. Arachidonic acid metabolism: role in inflammation. Zeitschrift fur Rheumatologie 50 Suppl 1, 3-6 (1991).

25 Zhou, Y. \& Danbolt, N. C. Glutamate as a neurotransmitter in the healthy brain. J Neural Transm (Vienna) 121, 799-817, doi:10.1007/s00702-014-1180-8 (2014).

26 Abbas, A.-K. et al. S-Sulfo-Cysteine is an Endogenous Amino Acid in Neonatal Rat Brain but an Unlikely Mediator of Cysteine Neurotoxicity. Neurochemical research 33, 301-307, doi:10.1007/s11064-007-9441-7 (2008).

27 Wu, G. \& Morris, S. M., Jr. Arginine metabolism: nitric oxide and beyond. The Biochemical journal 336 ( Pt 1), 1-17, doi:10.1042/bj3360001 (1998).

28 Ware, L. B. et al. Low plasma citrulline levels are associated with acute respiratory distress syndrome in patients with severe sepsis. Crit Care 17, R10-R10, doi:10.1186/cc11934 (2013).

29 Wijnands, K. A., Castermans, T. M., Hommen, M. P., Meesters, D. M. \& Poeze, M. Arginine and citrulline and the immune response in sepsis. Nutrients 7, 1426-1463, doi:10.3390/nu7031426 (2015).

30 Adusumilli, N. C., Zhang, D., Friedman, J. M. \& Friedman, A. J. Harnessing nitric oxide for preventing, limiting and treating the severe pulmonary consequences of COVID-19. Nitric oxide : biology and chemistry 103, 4-8, doi:10.1016/j.niox.2020.07.003 (2020).

31 Cai, Y., Guo, T., Wang, Y. \& Du, J. Glutamate Metabolism Regulates Immune Escape of Glioma. Madridge Journal of Immunology 2, 53-57 (2018).

32 Pacheco, R., Gallart, T., Lluis, C. \& Franco, R. Role of glutamate on T-cell mediated immunity. J Neuroimmunol 185, 9-19, doi:10.1016/j.jneuroim.2007.01.003 (2007).

33 Ganor, Y. \& Levite, M. in Nerve-Driven Immunity (ed M. Levite) (Springer, 2012).

34 Moore, H. M. et al. Biospecimen reporting for improved study quality (BRISQ). Cancer Cytopathol 119, 92-101, doi:10.1002/cncy.20147 (2011).

35 WHO R\&D Blueprint novel Coronavirus COVID-19 Therapeutic Trial Synopsis, $<$ https://www.who.int/blueprint/priority-diseases/key-action/COVID19 Treatment_Trial_Design_Master_Protocol_synopsis_Final_18022020.pdf.> (

36 Smith, C. A., Want, E. J., O'Maille, G., Abagyan, R. \& Siuzdak, G. XCMS: processing mass spectrometry data for metabolite profiling using nonlinear peak alignment, matching, and identification. Analytical chemistry 78, 779-787, doi:10.1021/ac051437y (2006).

37 Shen, X. et al. Normalization and integration of large-scale metabolomics data using support vector regression. Metabolomics : Official journal of the Metabolomic Society 12, 89, doi:10.1007/s11306-016-1026-5 (2016). 
medRxiv preprint doi: https://doi.org/10.1101/2020.09.06.20189159; this version posted September 8, 2020. The copyright holder for this preprint

(which was not certified by peer review) is the author/funder, who has granted medRxiv a license to display the preprint in perpetuity.

It is made available under a CC-BY-NC-ND 4.0 International license.

38 Shen, X. et al. Metabolic reaction network-based recursive metabolite annotation for untargeted metabolomics. Nature communications 10, 1516, doi:10.1038/s41467-01909550-x (2019).

39 Mukaka, M. M. Statistics corner: A guide to appropriate use of correlation coefficient in medical research. Malawi medical journal : the journal of Medical Association of Malawi 24, 69-71 (2012).

40 Struwe, W. et al. The COVID-19 MS Coalition-accelerating diagnostics, prognostics, and treatment. The Lancet 395, 1761-1762, doi:10.1016/S0140-6736(20)31211-3 (2020).

41 Sumner, L. W. et al. Proposed minimum reporting standards for chemical analysis Chemical Analysis Working Group (CAWG) Metabolomics Standards Initiative (MSI). Metabolomics : Official journal of the Metabolomic Society 3, 211-221, doi:10.1007/s11306-007-0082-2 (2007). 
medRxiv preprint doi: https://doi.org/10.1101/2020.09.06.20189159; this version posted September 8, 2020. The copyright holder for this preprint

(which was not certified by peer review) is the author/funder, who has granted medRxiv a license to display the preprint in perpetuity.

It is made available under a CC-BY-NC-ND 4.0 International license .

\section{Figures}

Fig. 1 Chord diagram of correlations between metabolites and immune markers in COVID-19 patients. Spearman correlations $>0.5$ or $<-0.5$ are displayed and with $\mathrm{p}<0.05$. 


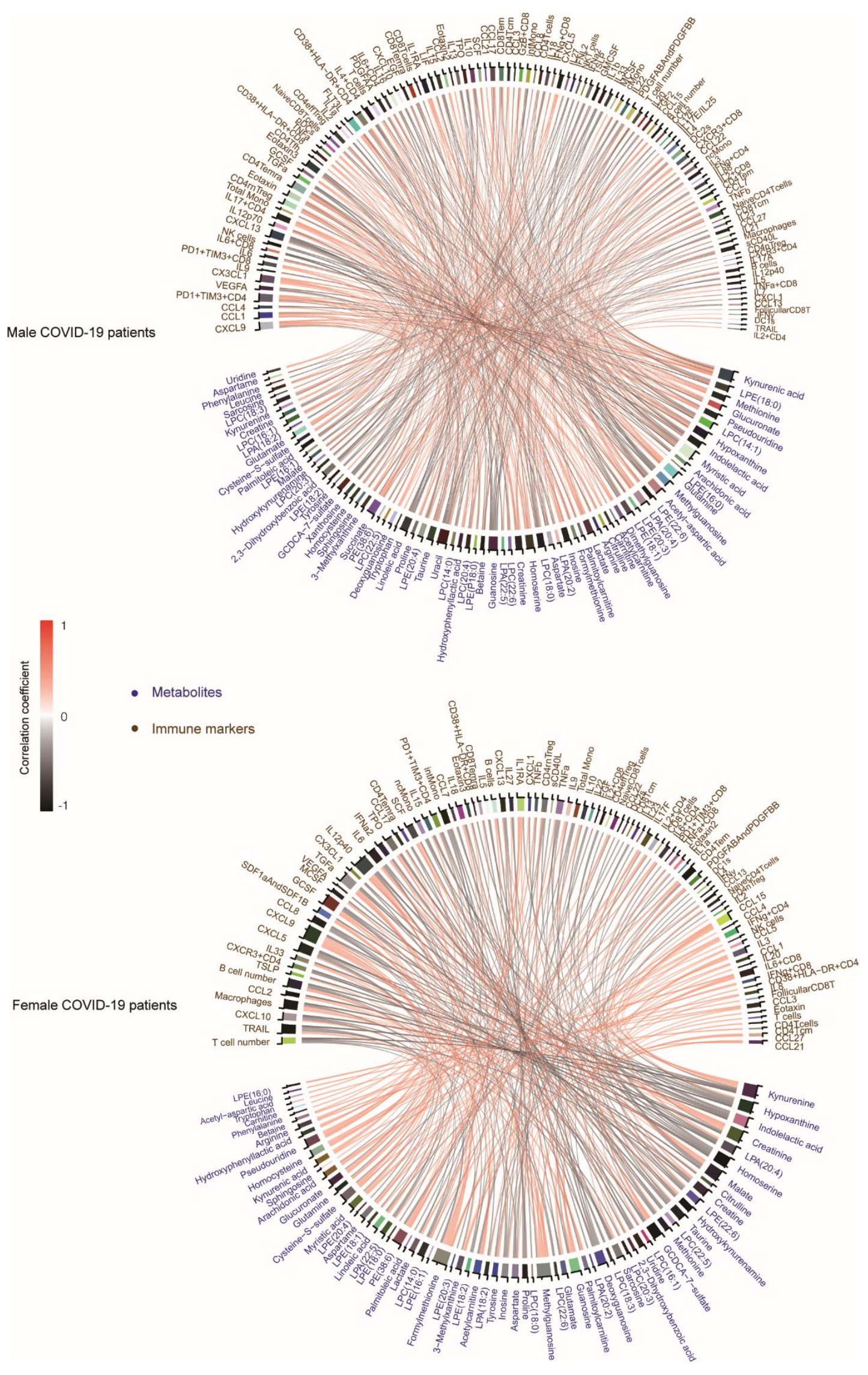


medRxiv preprint doi: https://doi.org/10.1101/2020.09.06.20189159; this version posted September 8, 2020. The copyright holder for this preprint

(which was not certified by peer review) is the author/funder, who has granted medRxiv a license to display the preprint in perpetuity.

It is made available under a CC-BY-NC-ND 4.0 International license.

Fig. 2 Tryptophan pathway metabolites and immune responses. a, Correlation between kynurenic acid (KA) and immune markers in males with COVID-19 (Pt. M, n=17) and females with COVID-19 (Pt. F, $\mathrm{n}=22$ ). $95 \%$ confidence intervals (CIs) for the correlation coefficients are indicated as shaded areas colored according to patient sex. b, Tryptophan (T) metabolism pathway schematic. c, Heatmap showing correlation between tryptophan metabolites and immune markers in males and females with COVID-19. Spearman correlations $>0.5$ or $<-0.5$ are displayed, $\mathrm{p}<0.05$. $\mathbf{d}$, Correlation between age and KA:kynurenine $(\mathrm{K})$ ratio in patients with COVID-19 and healthcare workers (HCWs). e, Correlation between KYAT3 (expression averaged within each age group) and age in Genotype-Tissue Expression (GTEx) samples (n=729 males, 1914 females). Metabolites are displayed as ion intensity log 10 transformed, cytokines and chemokines are $\mathrm{pg} / \mathrm{mL} \log 10$ transformed, $\mathrm{T}$ cell subsets are $\%$ in CD3 T cells, T cell number are $10^{\wedge} 6$ cells $/ \mathrm{mL}, \mathrm{PBMCs}$ are \% in live PBMCs. 

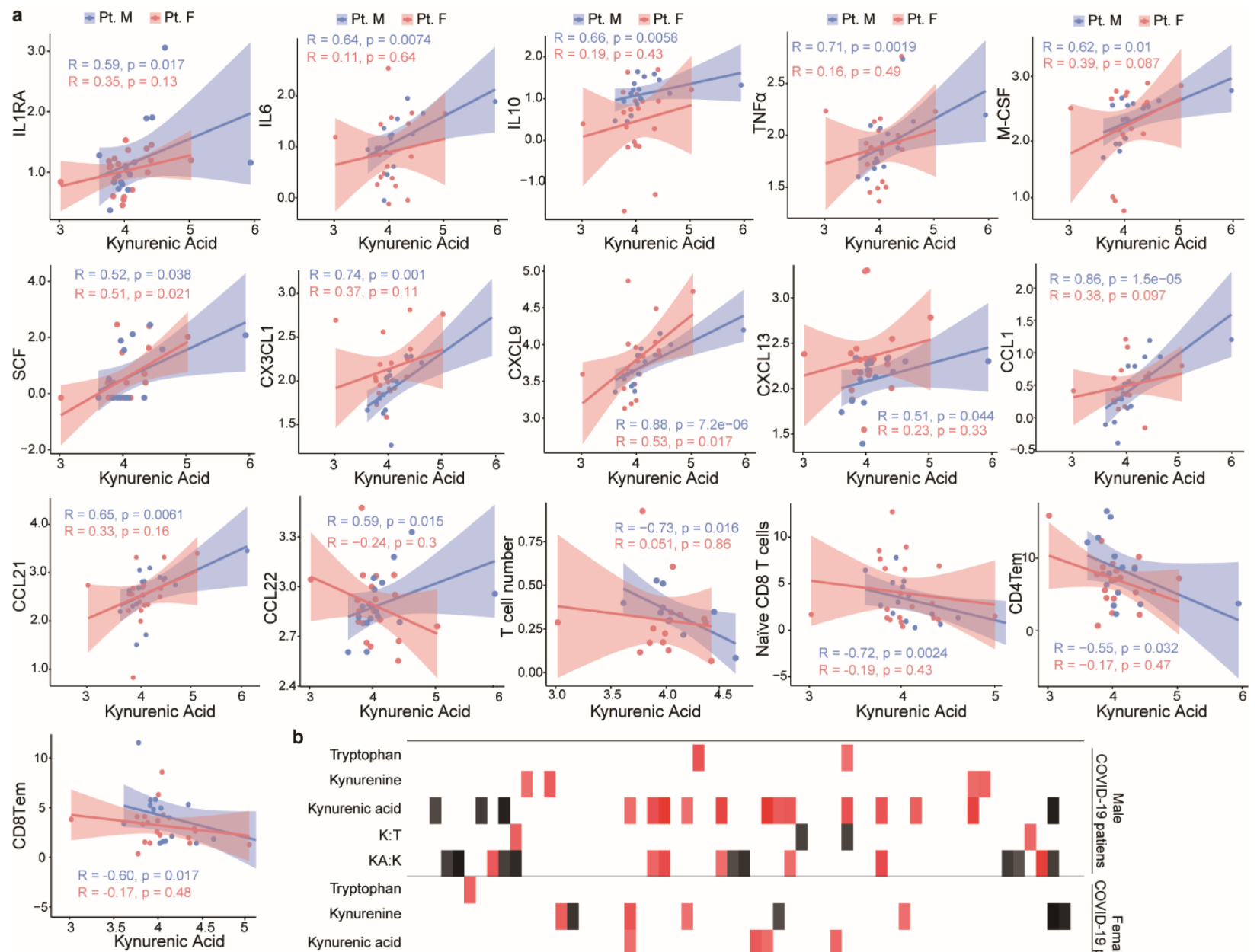

b

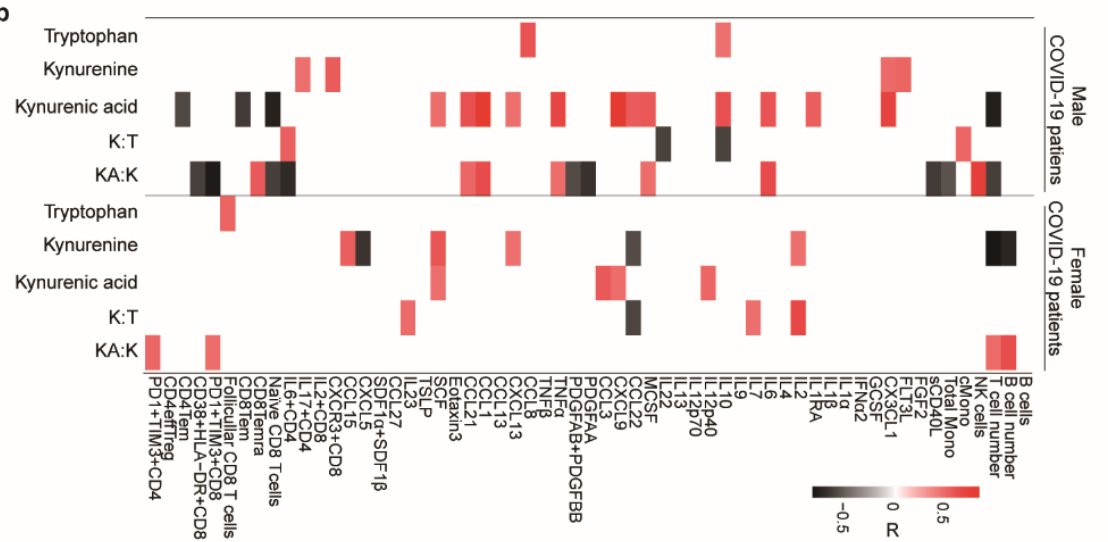

c

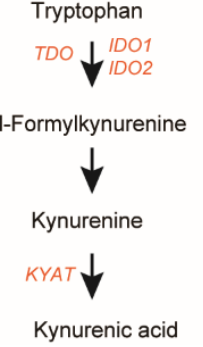

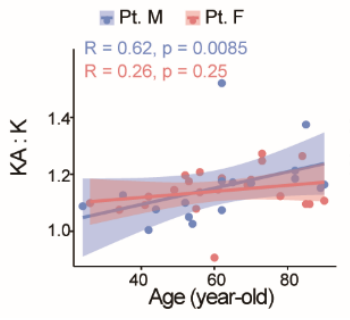
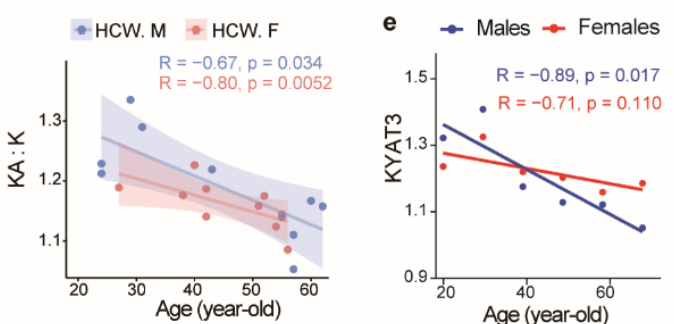
medRxiv preprint doi: https://doi.org/10.1101/2020.09.06.20189159; this version posted September 8, 2020. The copyright holder for this preprint

(which was not certified by peer review) is the author/funder, who has granted medRxiv a license to display the preprint in perpetuity.

It is made available under a CC-BY-NC-ND 4.0 International license .

Fig. 3 Tryptophan metabolites, immune markers and disease severity. a, Heatmap of correlations between metabolites in the tryptophan pathway and immune markers by disease severity. Spearman correlations $>0.5$ or $<-0.5$ are displayed with a $\mathrm{p}<0.05$. b. Correlation between kynurenic acid (KA) and immune markers by disease severity. $95 \%$ confidence intervals (CIs) for the correlation coefficients are indicated in shaded area colored according to disease progression status. c, Comparison of the ratio of KA:kynurenine (K) level by disease severity stratified by sex. Stabilized (females $n=16$, males =11), deteriorated (females $n=6$, males $n=6$ ). Nonparametric Kruskal-Wallis rank sum test with pairwise Wilcoxon Mann-Whitney $\mathrm{U}$ test, $p$ values adjusted for false discovery rate (Benjamini-Hochberg). ${ }^{* *} p<0.01$, NS. not significant. d, Correlation between the ratio of KA:kynurenine (K) and CXCL9 and CCL1 stratified by disease severity and sex. Metabolites are displayed as ion intensity log 10 transformed, cytokines and chemokines are $\mathrm{pg} / \mathrm{mL} \log 10$ transformed, $\mathrm{T}$ cell subsets are \% in CD3 T cells, $\mathrm{T}$ cell number are $10^{\wedge} 6$ cells $/ \mathrm{mL}$, PBMCs are $\%$ in live PBMCs. 
a

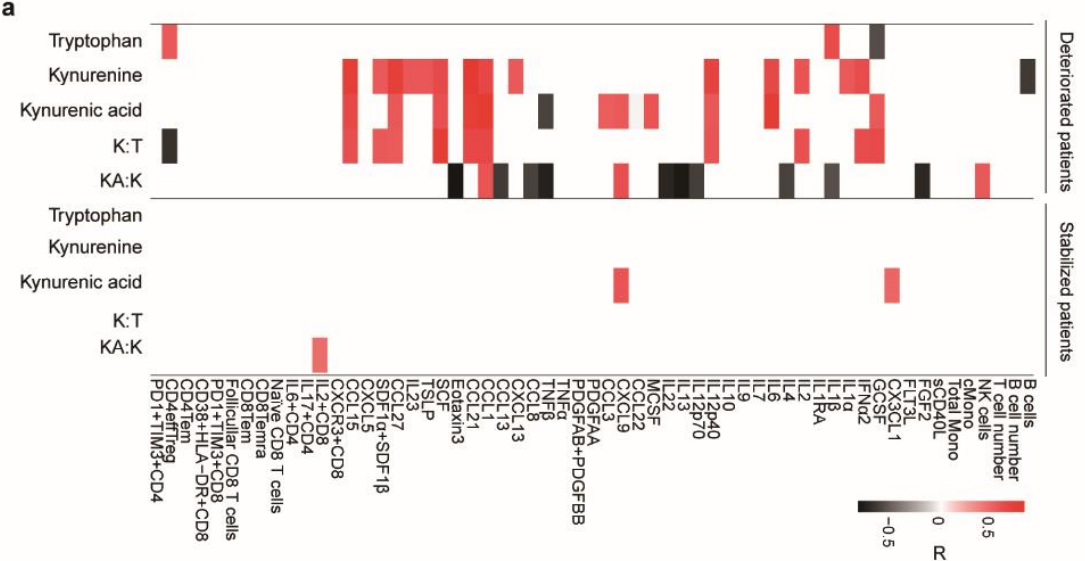

b $\Rightarrow$ Deteriorated $=$ Stabilized
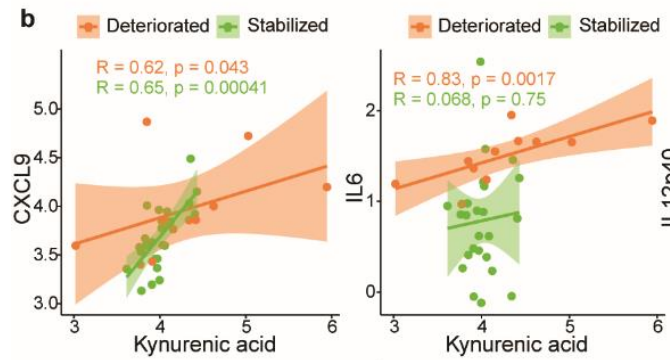

Deteriorated $=$ Stabilized

$F$ Deteriorated $=$ Stabilized
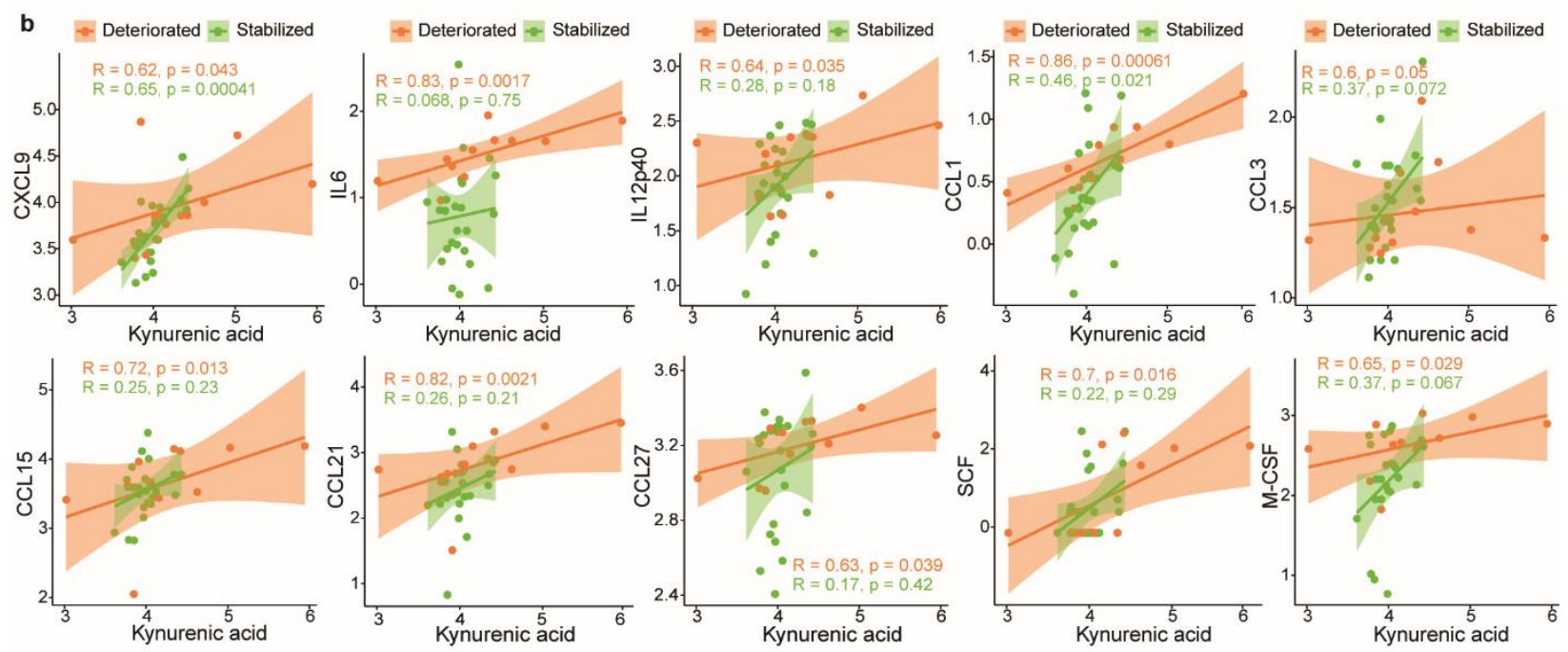

c
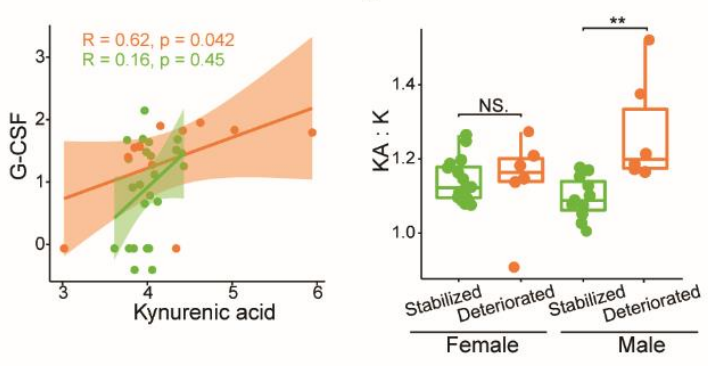

d Male COVID-19 patients
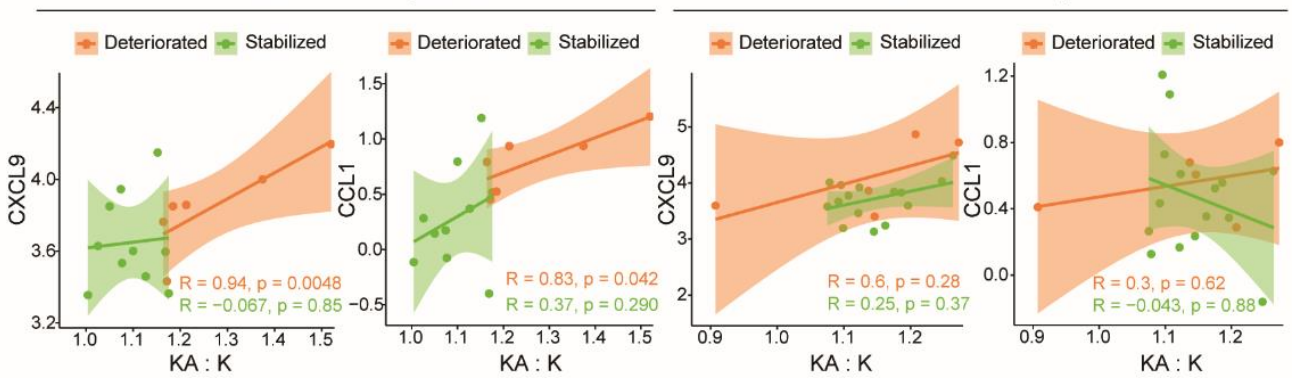
Fig. 4. Glutamate, immune markers and disease severity. a, Comparison of glutamate levels in stablized patients and deteriorated patients (left panel) and stratified by sex (right panel). Stabilized patients $(\mathrm{n}=27)$, deteriorated patients $(n=12)$, stabilized females $(n=16)$, deteriorated females $(n=6)$, stabilized males $(n=$ 11 ), and deteriorated males $(n=6)$. Nonparametric Kruskal-Wallis rank sum test with pairwise Wilcoxon Mann-Whitney $\mathrm{U}$ test, $p$ values adjusted for false discovery rates (FDR) (Benjamini-Hochberg). ** $p<0.01$, NS. not significant. b, Correlation between glutamate and Eotaxin2, IL5, IL6, CD4 T cells, CD4rnTreg cells, CD8 $\mathrm{T}$ cells and $\mathrm{GzB}+\mathrm{CD} 8$ cells in stabilized patients and deteriorated patients. c, Correlation between glutamate and immune markers eotaxin2, IL5, IL6, CD4 T cells, CD8 T cells, GzB+CD8 cells, and IL6 in stabilized patients and deteriorated patients stratified by sex. 95\% confidence intervals (CIs) for the correlation coefficients were indicated as the shadowed area colored according to progression status. Metabolites are displayed as ion intensity $\log 10$ transformed, cytokines and chemokines are $\mathrm{pg} / \mathrm{mL} \log 10$ transformed, $\mathrm{T}$ cell subsets are $\%$ in $\mathrm{CD} 3 \mathrm{~T}$ cells, $\mathrm{T}$ cell number are $10^{\wedge} 6$ cells $/ \mathrm{mL}, \mathrm{PBMCs}$ are $\%$ in live PBMCs. 

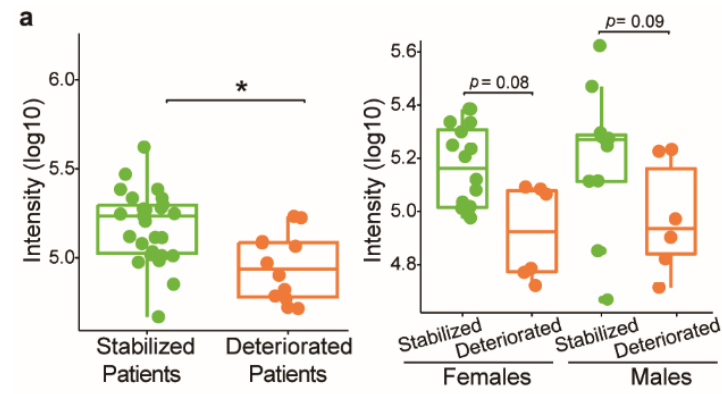

b $=$ Deteriorated $*$ Stabilized

$\rightarrow$ Deteriorated $*$ Stabilized
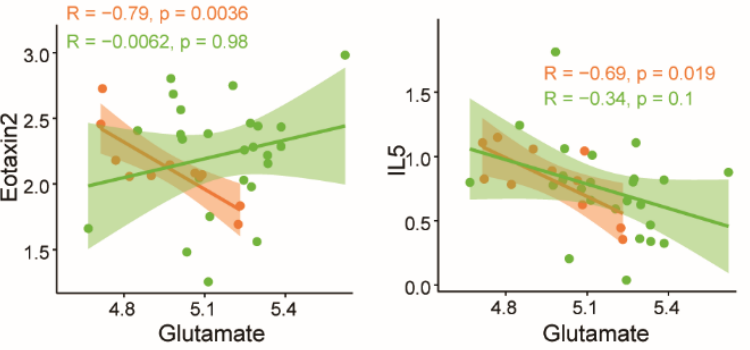

$\approx$ Deteriorated $\approx$ Stabilized
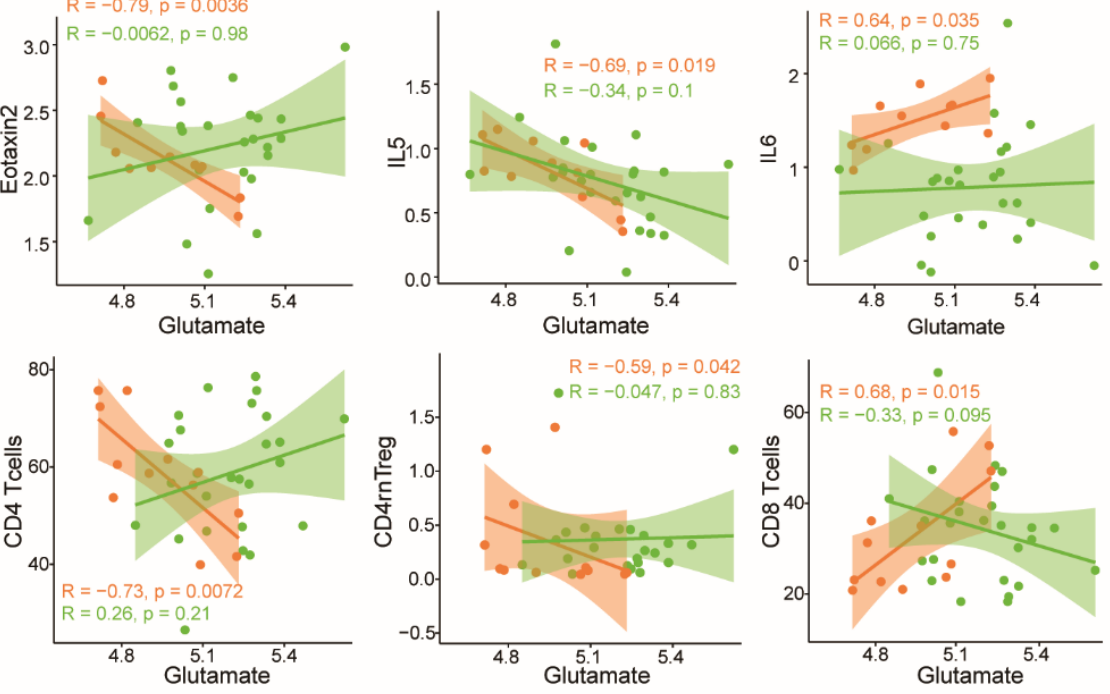

Male COVID-19 patients
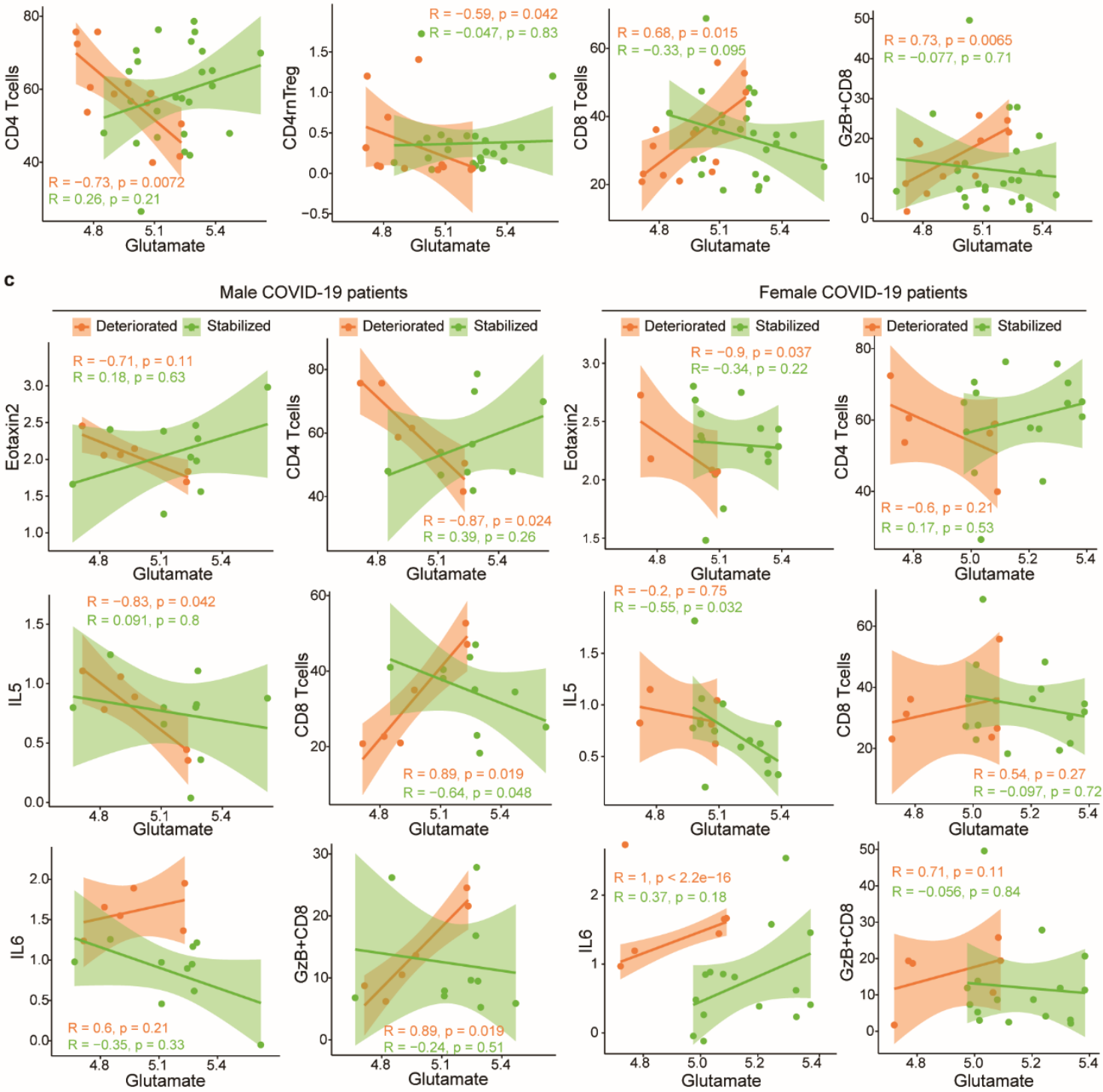


\section{Methods}

\section{Clinical biospecimens}

Serum samples were collected from patients enrolled in the IMPACT study from Cohort A as described and stored at $-20^{\circ} \mathrm{C}^{2}$. Cohort $\mathrm{A}$ consisted of 39 patients aged $\geq 18$ years old that tested positive for SARS-CoV-2 by RT-PCR from nasopharyngeal and/or oropharyngeal swabs (females $n=22$, males $n=17)^{34}$. Intersex individuals were not represented in this study. Prior to the serum collection, these patients were not in an intensive care unit, had not received tocilizumab and had not received high dose corticosteroids. Patients on hydroxychloroquine $(n=29)$ and remdesivir $(n=3)$ were not excluded. For control groups, we used 20 serum samples collected from COVID19 uninfected health care workers working at the Yale-New Haven Hospital between April $2^{\text {nd }}$ and April $28^{\text {th }} 2020$ who enrolled in the IMPACT study (females $n=10$, males $n=10$ ). The detailed demographics and clinical characteristics of these study participants and controls are shown in Extended Data Table 1.

\section{Immune markers and analysis of disease severity}

An immune panel of markers for each patient was obtained and published in a previous study ${ }^{2}$. The patients were assessed with a locally developed clinical scoring system for disease severity ${ }^{17}$; 1: admitted and observed without supplemental oxygen, 2 : required $\leq 3 \mathrm{~L}$ supplemental oxygen via nasal canal to maintain $\mathrm{SpO} 2>92 \%$, 3: received tocilizumab, which per hospital treatment protocol required that the patient to require $>3 \mathrm{~L}$ supplemental oxygen to maintain $\mathrm{SpO} 2>92 \%$, or, required $>2 \mathrm{~L}$ supplemental oxygen to maintain $\mathrm{SpO} 2>92 \%$ and had a high sensitivity $\mathrm{C}$ reactive protein $(\mathrm{CRP})>70.4$ : the patient required intensive care unit (ICU) level care, 5: the patient required intubation and mechanical ventilation. In relation to the WHO scoring, our clinical score $1,2 / 3,4,5$ largely correspond to WHO score 3, 4, 5, 6/7, respectively ${ }^{35}$. Detailed demographic information is available from ${ }^{2}$. For the patients who are 90-year-old or older, their ages were protected health information, and 90 was put as the surrogate value for the analyses. Individuals with active chemotherapy against cancers, pregnant patients, patients with background hematological abnormalities, patients with autoimmune diseases and patients with a history of organ transplantation and on immunosuppressive agents, were excluded from this study. 


\section{Serum metabolite extraction}

Serum samples $(50 \mu \mathrm{L})$ were thawed and deactivated for COVID-19 in $150 \mu \mathrm{L}$ acetone:methanol $(50: 50 \mathrm{v} / \mathrm{v})$ for $60 \mathrm{~min}$ at room temperature. Control samples were treated in the exactly the same manner. To precipitate proteins, the samples were incubated for 2 hours at $-20{ }^{\circ} \mathrm{C}$, followed by centrifugation at $13,000 \mathrm{rpm}(15,000 \times \mathrm{g})$ and $4{ }^{\circ} \mathrm{C}$ for $15 \mathrm{~min}$. The resulting supernatant was removed and evaporated to dryness for $12 \mathrm{~h}$ using a vacuum concentrator (Thermo Fisher Scientific, Waltham, MA, USA). The dry extracts were then reconstituted in $100 \mu \mathrm{L}$ of $\mathrm{ACN}: \mathrm{H}_{2} \mathrm{O}$ $(1: 1, \mathrm{v} / \mathrm{v})$, sonicated for $10 \mathrm{~min}$, and centrifuged at $13,000 \mathrm{rpm}(15,000 \times \mathrm{g})$ and $4{ }^{\circ} \mathrm{C}$ for $15 \mathrm{~min}$,

to remove insoluble debris. The supernatants were transferred to ultra performance liquid chromatography (UPLC) autosampler vials (Thermo Scientific, Waltham, MA, USA). A pooled quality control (QC) sample was prepared by mixing $5 \mu \mathrm{L}$ of extracted solution from each sample into a similar UPLC vial. All the vials were then capped and stored at $-80{ }^{\circ} \mathrm{C}$ prior to UPLC-mass spectrometry (MS) analysis.

\section{UPLC-MS-based metabolomics analysis}

To comprehensively analyze the serum metabolome, both hydrophilic interaction chromatography (HILIC)-MS and reverse phase liquid chromatography (RPLC)-MS approaches were used. A UPLC system (H-Class ACQUITY, Waters Corporation, Milford, MA, USA), coupled to a quadrupole time-of flight (QTOF) (Xevo G2-XS QTOF, Waters Corporation, Milford, MA, USA), was used for MS data acquisition. A Waters ACQUITY UPLC BEH Amide column (particle size, $1.7 \mu \mathrm{m} ; 100 \mathrm{~mm}$ (length) $\times 2.1 \mathrm{~mm}$ (i.d.)) and Waters ACQUITY UPLC BEH C18 column (particle size, $1.7 \mu \mathrm{m} ; 100 \mathrm{~mm}$ (length) $\times 2.1 \mathrm{~mm}$ (i.d.)) were used for the UPLC-based separation of metabolites. The column temperature was kept at $25^{\circ} \mathrm{C}$ for HILIC-MS analysis and $30{ }^{\circ} \mathrm{C}$ for RPLC-MS analysis. The solvent flow rate was $0.5 \mathrm{~mL} / \mathrm{min}$, and the sample injection volume was $4 \mu \mathrm{L}$ for HILIC-MS and RPLC in positive mode analysis, $2 \mu \mathrm{L}$ for HILIC-MS in negative mode, and $6 \mu \mathrm{L}$ for RPLC-MS negative mode. For HILIC-MS analysis, mobile phase A was $25 \mathrm{mM}$ $\mathrm{NH}_{4} \mathrm{OH}$ and $25 \mathrm{mM} \mathrm{NH} \mathrm{NHAc}_{4} \mathrm{On}$ water, while the mobile phase $\mathrm{B}$ was acetonitrile, for both electrospray ionization (ESI), positive and negative mode, respectively. The linear gradient was set as follows: 0 0.5 min: $95 \% \mathrm{~B}$; $0.5 \sim 7 \mathrm{~min}: 95 \% \mathrm{~B}$ to $65 \% \mathrm{~B} ; 7 \sim 8 \mathrm{~min}: 65 \% \mathrm{~B}$ to $40 \% \mathrm{~B} ; 8 \sim 9$ min: 40\% B; 9 9.1 min: 40\% B to 95\% B; 9.1 12 min: 95\% B. For RPLC-MS analysis, the mobile 
phases $A$ was $0.1 \%$ formic acid in $\mathrm{H}_{2} \mathrm{O}$, while the mobile phases $\mathrm{B}$ was $0.1 \%$ formic acid in acetonitrile, respectively, for ESI+. Mobile phase A was $5 \mathrm{mM} \mathrm{NH}_{4} \mathrm{OAc}$ in $\mathrm{H}_{2} \mathrm{O}$, while the mobile phases B was acetonitrile for ESI-. The linear gradient was set as follows: 0 1 min: 1\% B; 1 8 min: $1 \%$ B to $100 \% \mathrm{~B}$; 8 10 min: $100 \% \mathrm{~B} ; 10 \sim 10.1 \mathrm{~min}: 100 \% \mathrm{~B}$ to $1 \% \mathrm{~B} ; 10.1 \sim 12 \mathrm{~min}: 1 \% \mathrm{~B}$. Pooled samples were analyzed every eight injections during the UPLC-MS analysis to monitor the stability of the data acquisition and used for subsequent data normalization.

QTOF scan data (300 ms/scan; mass scan range 50-1000 Da) were initially acquired for each biological sample for metabolite quantification. Then, both DDA (data-dependent acquisition) data (QTOF scan time: $100 \mathrm{~ms} / \mathrm{scan}$, MSMS scan time $500 \mathrm{~ms} / \mathrm{scan}$, collision energy $20 \mathrm{eV}$, top 5 most intense ions were selected for fragmentation, exclude former target ions (4 s after 2 occurrences)) and $\mathrm{MS}^{\mathrm{E}}$ data (low energy scan: $300 \mathrm{~ms} / \mathrm{scan}$, collision energy $6 \mathrm{eV}$; high energy scan: 300 $\mathrm{ms} / \mathrm{scan}$, collision energy $20 \mathrm{eV}$, mass scan range 25-1000 Da) were acquired for QC samples to enable metabolite identification. ESI source parameters on the Xevo GS-XS QTOF were set as the following: capillary voltage $1.8 \mathrm{kV}$, sampling cone $30 \mathrm{~V}$, source temperature $100{ }^{\circ} \mathrm{C}$, desolvation temperature $550{ }^{\circ} \mathrm{C}$, cone gas flow $40 \mathrm{~L} / \mathrm{h}$, desolvation gas flow $900 \mathrm{~L} / \mathrm{h}$.

\section{UPLC-MS data processing}

The raw MS data (.raw) were converted to mzML files using ProteoWizard MSConvert (version 3.0.6150, www.proteowizard.sourceforge.net/). The parameters of min SNR and min peak spacing were set as 0.1 for peak picking in ProteoWizard. The files were then processed in $\mathrm{R}$ (version 3.4.3), using the XCMS package for feature detection, retention time correction, and alignment ${ }^{36}$. The XCMS processing parameters were optimized and set as follows: mass accuracy for peak detection $=20$ ppm; peak width $\mathrm{c}=(2,30)$; snthresh $=6 ; \mathrm{bw}=10 ;$ mzwid $=0.015 ;$ minfrac $=0.5$. The CAMERA package was used for subsequent peak annotation. The resulting data were normalized using the support vector regression algorithm in $\mathrm{R}$, to remove an unwanted system error that occurred among intra- and inter-batches ${ }^{37}$. Initial metabolite identification was performed using the MetDNA algorithm ${ }^{38}$. Metabolites were further identified by matching retention time with an in-house metabolite standard library. In addition, metabolite identification was carried out by matching accurate mass and experimental MS/MS data against online databases (METLIN and HMDB). 


\section{Multivariable logistic regression}

Multivariable logistic regression analyses were performed on the R platform (version 3.4.3) using an R function "glm ()" The model for each metabolite was adjusted for age, BMI and sex to discover metabolites associated with COVID-19 disease. Levels of metabolites are $\log 10$ transformed ion intensity. $p$ values were adjusted for multiple testing with Benjamini-Hochbergbased FDR using an R function "p.adjust ()".

\section{Spearman correlation analysis}

Spearman correlation analyses were performed on R platform (version 4.0.2) using an $\mathrm{R}$ package "psych". Correlation coefficient $\mathrm{R}$ and $p$ values were calculated using an R function "corr.test ()" Using previously defined interpretations of correlation coefficients, we used an $|R|$ value of 0.5 1.0 to mark moderate-to-very high correlations ${ }^{39}$. Heatmaps were plotted using an $\mathrm{R}$ package "pheatmap".

\section{Chord diagram}

The chord diagrams were plotted on R platform (version 4.0.2) using an R package "circlize". Correlations between metabolites and immune responses with $\mathrm{R}>0.5$ or $<-0.5$, and $p$ value $<0.05$ were plotted out.

\section{Gene expression analysis}

Gene TPMs, subject phenotypes, and sample attributes data were downloaded from GTEX Portal (gtexportal.org, accession phs000424.v8.p2). After TPM values were transformed as $\log 10(\mathrm{TPM}+1)$, composite expression scores were calculated by adding individual expression values together. Patients who were 60 years or older were coded as "Older," while patients 30 years or younger were coded as "Young." After loading the expression data into R with the CePa package, Pearson correlation coefficients were calculated for pairs of target genes within each tissue of each sex, and data was visualized as a heatmap displaying the difference between male and female coefficients using the ComplexHeatmap package. Male-specific correlations were validated by scatter plots and linear regressions, which were generated using the ggplot2 R package. 


\section{Data Availability and Code Availability}

Untargeted metabolomics data, metabolomics protocols, and code is available on the $\begin{array}{lllll}\text { MetaboLights data repository accession } & \text { number } & \text { MTBLS1987 }\end{array}$ (https://www.ebi.ac.uk/metabolights/). Clinical and immunological data is available from previous publication $^{2}$. Data processing R code is available in Supplementary Information.

\section{Ethical statements}

This study was approved by Yale Human Research Protection Program Institutional Review Boards (FWA00002571, Protocol ID. 2000027690). Informed consent was obtained from all enrolled patients and healthcare workers.

\section{Acknowledgements}

We gratefully acknowledge the study participants for their time and commitment to the study. We thank all members of the clinical team at Yale-New Haven Hospital for their dedication and work which made this study possible. This work was in part supported by a gift from the Yale University Rapid Relief Fund, Women's Health Research at Yale Pilot Project Program, Fast Grant from Emergent Ventures at the Mercatus Center, Mathers Foundation, the Beatrice Kleinberg Neuwirth Fund, and the Ludwig Family Foundation. IMPACT received support from the Yale COVID-19 Research Resource Fund. A.I. is an Investigator of the Howard Hughes Medical Institute. D.J.K. was a Paul and Daisy Soros Fellow and was supported by a grant from the National Cancer Institute (NCI) of the National Institutes of Health (NIH) (F30CA236466) and by a MSTP training grant from the NIH (T32GM007205, T32GM136651). S.A.K was supported by CTSA Grant Number UL1TR001863 from the National Center for Advancing Translational Science (NCATS), components of the NIH, and NIH roadmap for Medical Research, and the Lampman Research Fund in Yale Surgical Oncology. Support was also provided by the Beatrice Kleinberg Neuwirth Fund, Yale Schools of Public Health and Medicine, and NIH U19 AI08992 awarded to A.I.K.

\section{Author Contributions}

All authors contributed to the data discussions and writing of this manuscript. Additional contributions: Y.C carried out the metabolomics mass spectrometry and data analysis, and 
prepared figures and tables, D.J.K carried out design of the gene expression analysis, performed the gene expression analysis and interpreted the results, T.T was involved in acquiring the biospecimens and experimental design, D.B validated statistical analysis design and reproduced the data independently, S.M. assisted with the biostatistics in the manuscript, N.J.W.R was involved in experimental design and figure design, A.C-M, A.J.M, M.C.M acquired the biospecimens and performed viral inactivation, B.I, J.K, C.L, T.M, J.O, J.S, P.W acquired immune cell data, A.I.K, contributed to data and clinical interpretation, S.A.K contributed to data interpretation and clinical discussions on data, A.I was involved in acquiring the biospecimens and experimental design, securing funds and supervising the study, C.H.J was involved in experiment design, data analysis, drafting the initial manuscript, securing funds and supervising the study. Y.C., D.B., C.H.J., and N.J.W.R are members of the COVID-19 Mass Spectrometry Coalition ${ }^{40}$.

\section{Yale IMPACT Research Team authors (in alphabetical order)}

Tara Alpert, Kelly Anastasio, Michael H. Askenase, Maria Batsu, Santos Bermejo, Sean Bickerton, Anderson Brito, Kristina Brower, Molly L. Bucklin, Staci Cahill, Melissa Campbell, Yiyun Cao, Edward Courchaine, Rupak Datta, Giuseppe Deluliis, Charles Dela Cruz, Rebecca Earnest, Shelli Farhadian, Joseph Fauver, Renata Filler, John Fournier, Bertie Geng, Laura Glick, Nathan Grubaugh, Ryan Handoko, Christina Harden, Cole Jensen, Chaney Kalinich, William Khoury-Hanold, Lynda Knaggs, Maxine Kuang, Eriko Kudo, Sarah Lapidus, Joseph Lim, Melissa Linehan, Feimei Liu, Peiwen Lu, Alice Lu-Culligan, Maksym Minasyan, Amyn A. Malik, Anjelica Martin, Irene Matos, David McDonald, Maura Nakahata, Nida Naushad, Allison Nelson, Jessica Nouws, Angela Nuñez, Marcella Nunez-Smith, Abeer Obaid, Camila Odio, Ji Eun Oh, Saad B. Omer, Isabel M. Ott, Annsea Park, Hong-Jai Park, Xiaohua Peng, Mary Petrone, Sarah Prophet, Harold Rahming. Tyler Rice, Aaron Ring, Kadi-Ann Rose, Lorenzo Sewanan, Lokesh Sharma, Albert Shaw, Denise Shepard, Erin Silva, Mikhail Smolgovsky, Eric Song, Nicole Sonnert, Yvette Strong, Codruta Todeasa, Maria Tokuyama, Jordan Valdez, Sofia Velazquez, Arvind Venkataraman, Pavithra Vijayakumar, Chantal B.F. Vogels, Eric Y. Wang, Annie Watkins, Elizabeth B. White, Anne L. Wyllie, Yexin Yang.

\section{Competing interest declaration}

The authors declare no competing financial or non-financial interests. 
medRxiv preprint doi: https://doi.org/10.1101/2020.09.06.20189159; this version posted September 8, 2020. The copyright holder for this preprint

(which was not certified by peer review) is the author/funder, who has granted medRxiv a license to display the preprint in perpetuity.

It is made available under a CC-BY-NC-ND 4.0 International license .

\section{Additional Information}

\section{Supplementary Information}

This file contains Supplementary Table 1. Correlations between metabolites and immune markers in patients with COVID-19. Supplementary Table 2. Correlations between metabolites and immune markers in healthcare workers. The two tables include the calculated spearman correlation coefficient and $p$ value. Supplementary Information contains data processing code for use in $\mathrm{R}$. 


\section{Extended Data Figures}
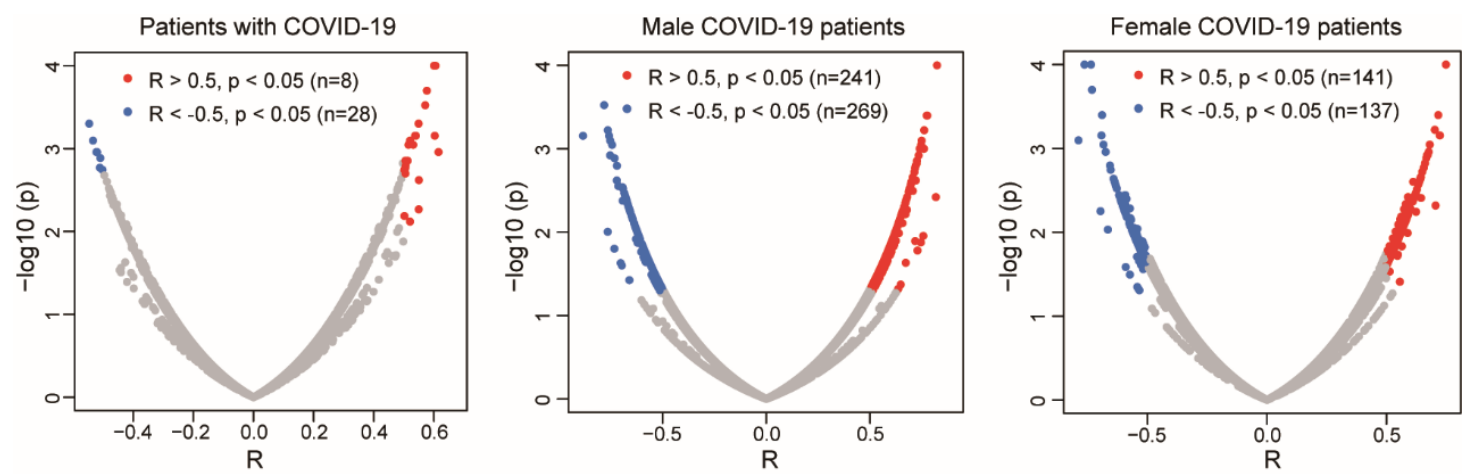

Extended Data Figure 1. Scatter plots of correlation coefficient against -log10 ( $p$ value) between metabolites and immune markers in all patients with COVID-19, males with COVID-19, and females with COVID-19, respectively. 

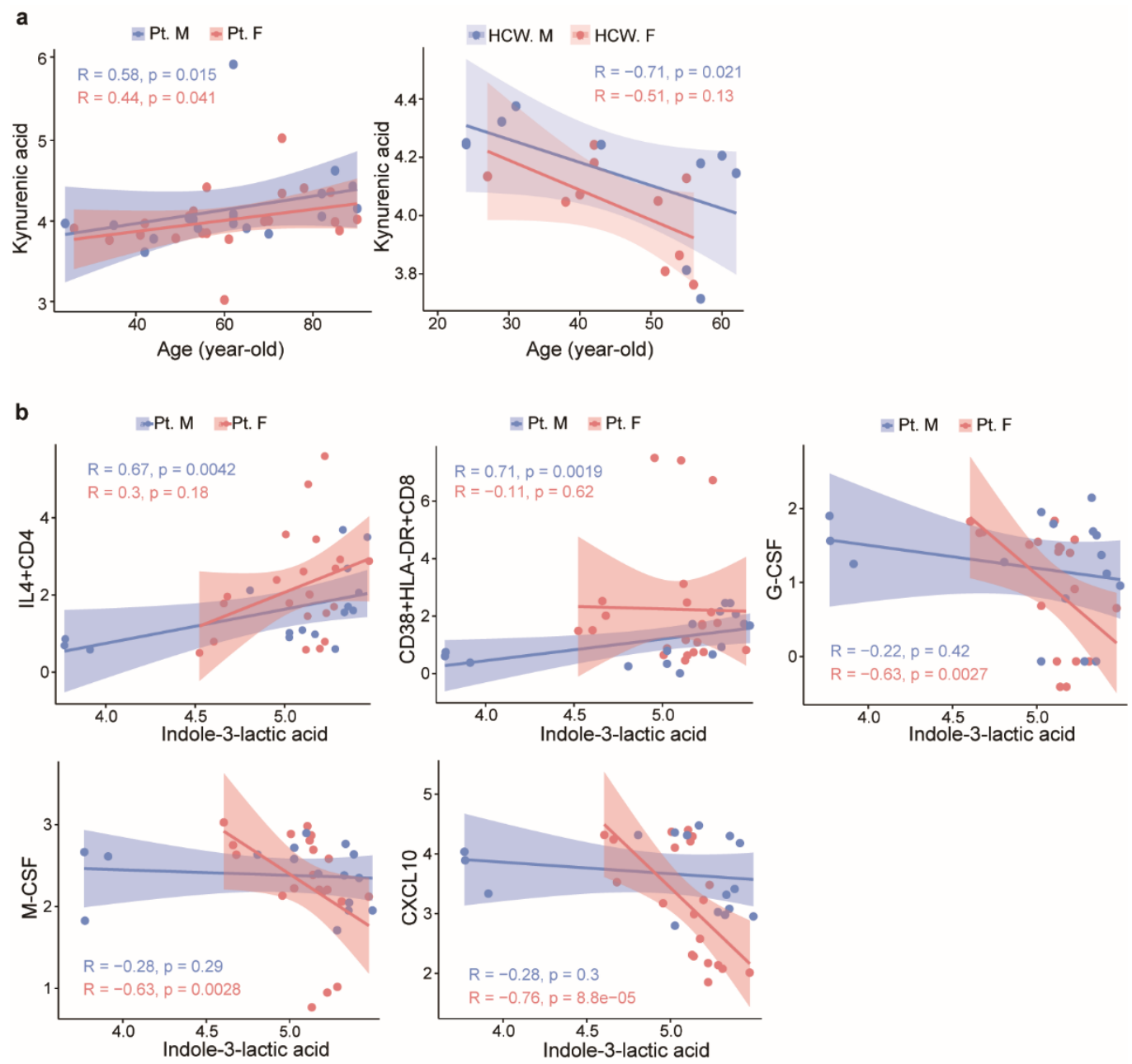

Extended Data Figure 2. Correlations between metabolites and immune markers in patients with COVID-19 and healthcare workers stratified by sex. a, Correlation between age and kynurenic acid levels in patients with COVID-19 (left) and HCWs (right). b, Correlation between indole-3-lactic acid and IL4+CD4, CD38+HLA-DR+CD8, G-CSF, M-CSF and CXCL10 in males with COVID-19 and females with COVID-19, respectively. 95\% confidence intervals (CIs) for the correlation coefficients were indicated as the shadowed area colored according to sex. 

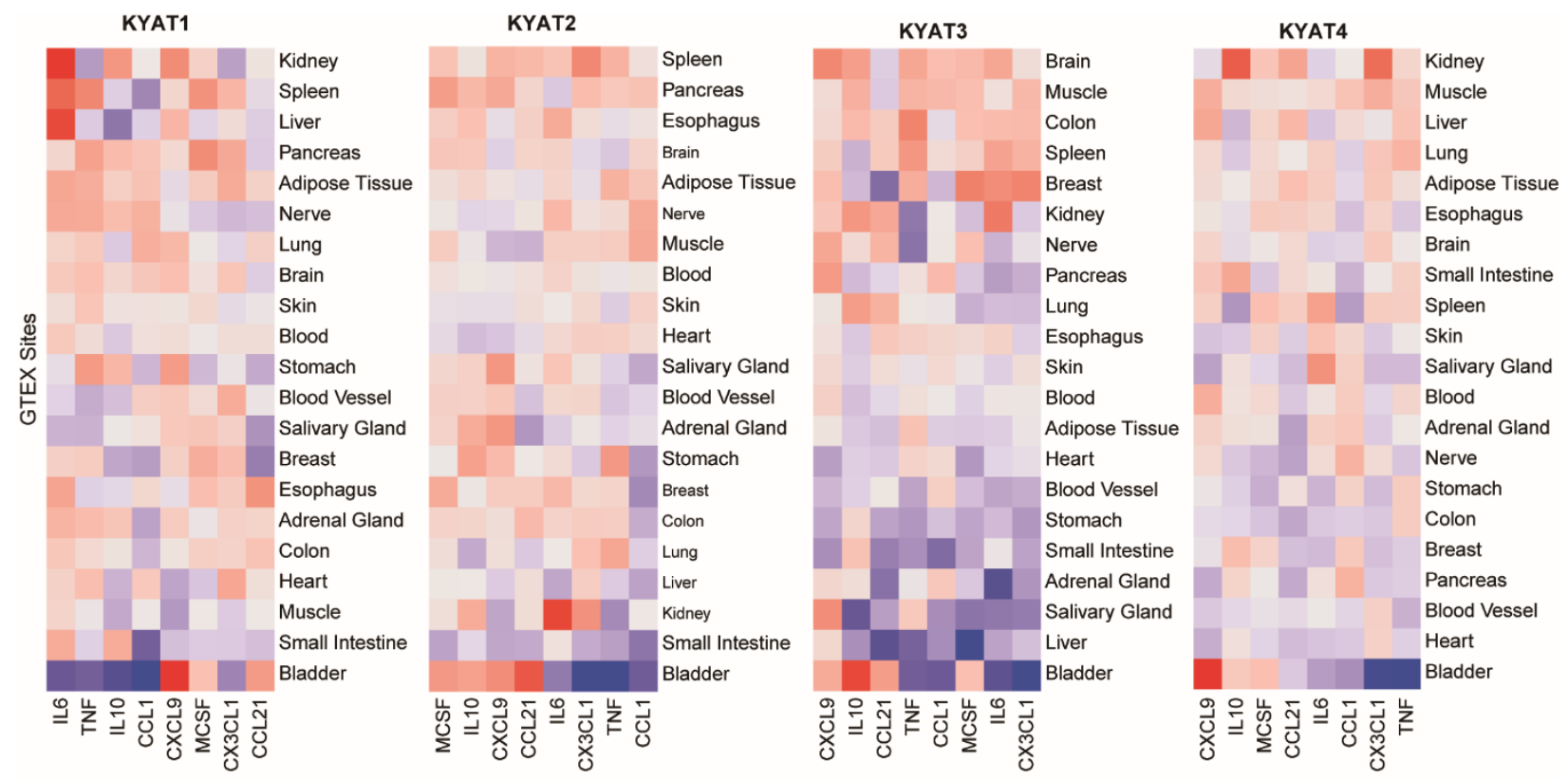

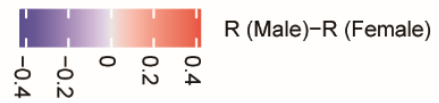

Extended Data Figure 3. Correlations between KYAT gene expression and cytokines positively associated with either high KA or KA:K. Pearson correlation coefficients were calculated for gene pairs within the indicated tissue for each sex using GTEx data. Differences in the correlations $\left(\mathrm{R}_{\text {Male }}-\mathrm{R}_{\mathrm{Female}}\right)$ are presented as heatmaps, with red indicating a more positive correlation in males and blue indicating a more positive correlation in females ( $\mathrm{n}=729$ males, 1914 females). 

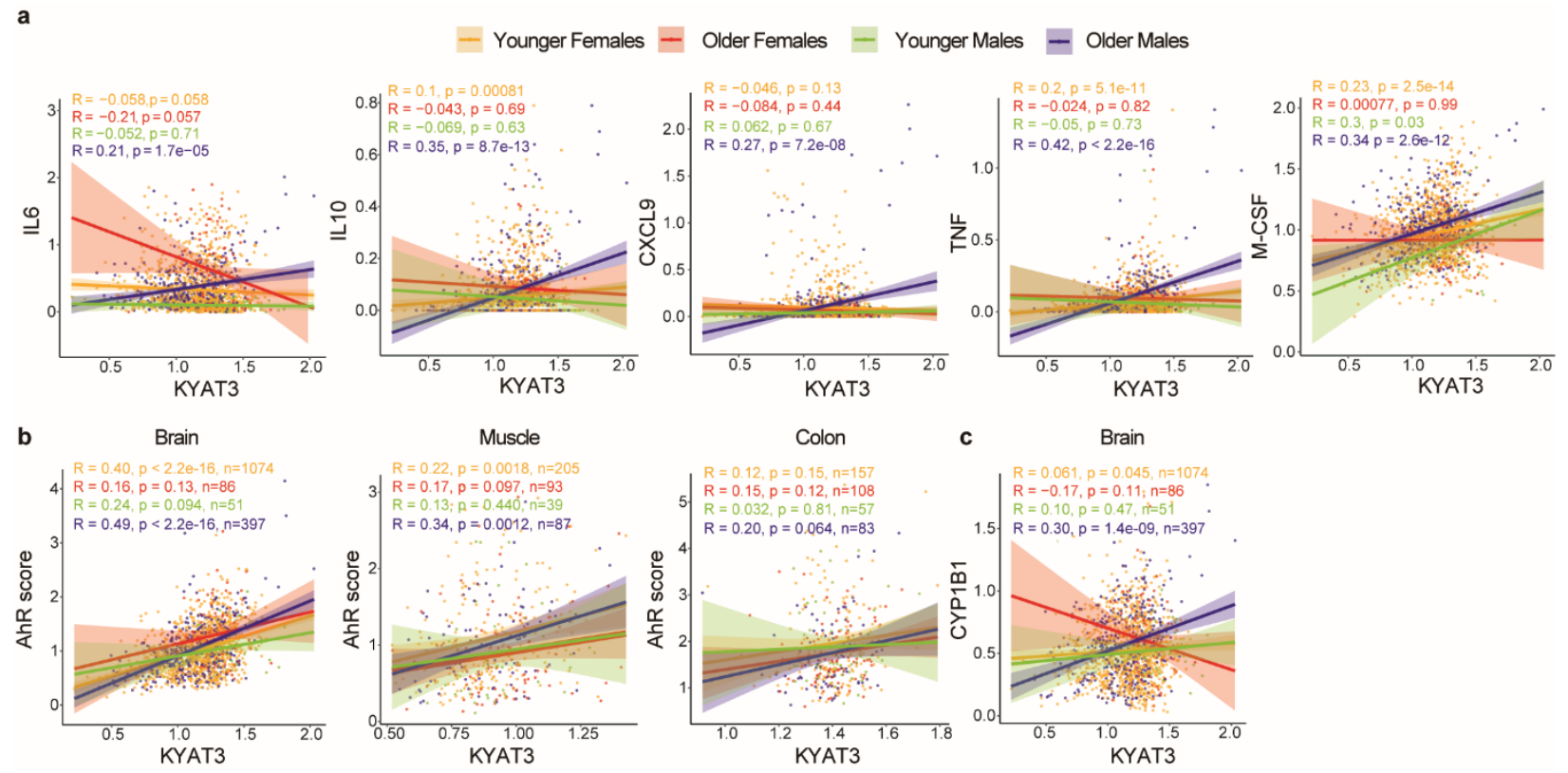

Extended Data Figure 4. Correlations between KYAT3, immune markers, and AhR activation in younger and older individuals, stratified by sex. a, Correlations between KYAT3 expression and IL6, IL10, CXCL9, TNF, and M-CSF in GTEx brain samples. b, Correlations between KYAT3 and AhR activation score in brain, muscle and colon. c, Correlation between KYAT3 and classic AhR target gene CYP1B1 in brain. 


\section{Extended Data table titles and footnotes}

Extended Data Table 1. Demographic and clinical characteristics of patients with COVID-19 and healthcare workers

\begin{tabular}{|c|c|c|c|c|}
\hline & \multicolumn{2}{|c|}{ Patients with COVID-19 $(n=39)$} & \multicolumn{2}{|c|}{ Healthcare workers $(n=20)$} \\
\hline & Female $\mathrm{n}(\%)$ & Male n (\%) & Female $\mathrm{n}(\%)$ & Male n (\%) \\
\hline Total & $22(56)$ & $17(44)$ & $10(50)$ & $10(50)$ \\
\hline \multicolumn{5}{|l|}{ Ethnicity ${ }^{*}$} \\
\hline Black/African American & 4 & 5 & 0 & 1 \\
\hline White & 13 & 9 & 10 & 7 \\
\hline Hispanic & 4 & 2 & 0 & 1 \\
\hline \multicolumn{5}{|l|}{ BMI $^{*}$} \\
\hline$<18$ & 1 & 0 & 0 & 0 \\
\hline $18-24.9$ & 6 & 4 & 3 & 3 \\
\hline $25-29.9$ & 5 & 7 & 4 & 2 \\
\hline $30-34.9$ & 8 & 3 & 0 & 3 \\
\hline$\geq 35$ & 2 & 3 & 3 & 2 \\
\hline Age - mean (SD) & $60.2(16.6)$ & $59.2(17.8)$ & $45.7(8.9)^{\S}$ & $44.2(14.9)^{\S}$ \\
\hline Days from symptom onset - mean (SD) & $12.3(9.0)$ & $9.2(5.5)$ & $\mathrm{N} / \mathrm{A}$ & $\mathrm{N} / \mathrm{A}$ \\
\hline Clinical Score - mean (SD) & $1.27(0.46)$ & $1.47(0.62)$ & $\mathrm{N} / \mathrm{A}$ & $\mathrm{N} / \mathrm{A}$ \\
\hline On Hydroxychloroquine $†$ & $18(81.8)$ & $11(64.7)$ & $\mathrm{N} / \mathrm{A}$ & $\mathrm{N} / \mathrm{A}$ \\
\hline On Remdesivir & $2(9.1)$ & $1(5.9)$ & $\mathrm{N} / \mathrm{A}$ & $\mathrm{N} / \mathrm{A}$ \\
\hline
\end{tabular}

N/A, not available for the data

*Data included when available $\dagger$ Status at first sample collection $\S$ Student's $t$-test, $p<0.05$, comparing with patients with COVID-19 
Extended Data Table 2. Positively identified metabolites from both COVID-19 patients and healthcare workers.

\begin{tabular}{|c|c|c|c|c|c|c|c|c|c|}
\hline Name & $m / z$ & $\begin{array}{c}\mathrm{RT} \\
\text { (seconds) }\end{array}$ & $\begin{array}{c}\text { Analysis } \\
\text { mode }^{*}\end{array}$ & MSI & Name & $m / z$ & $\begin{array}{c}\mathrm{RT} \\
\text { (seconds) } \\
\end{array}$ & $\begin{array}{c}\text { Analysis } \\
\text { mode }\end{array}$ & MSI \\
\hline Homoserine & 84.0445 & 351.66 & HILIC (+) & 1 & Uridine & 245.0760 & 150.07 & HILIC (+) & 1 \\
\hline Lactate & 89.0230 & 209.01 & HILIC (-) & 1 & Palmitoleic acid & 253.2169 & 430.09 & RPLC (-) & 1 \\
\hline Sarcosine & 90.0560 & 324.69 & HILIC (+) & 1 & Inosine & 267.0725 & 198.95 & HILIC (-) & 1 \\
\hline Uracil & 111.0194 & 82.96 & HILIC (-) & 1 & Aspartame & 275.1031 & 172.78 & HILIC (-) & 1 \\
\hline Creatinine & 112.0511 & 158.32 & HILIC (-) & 1 & Guanosine & 282.0830 & 242.79 & HILIC (-) & 1 \\
\hline Proline & 116.0703 & 286.25 & HILIC (+) & 1 & Xanthosine & 283.0677 & 294.85 & HILIC (-) & 1 \\
\hline Succinate & 117.0179 & 374.18 & HILIC (-) & 1 & Deoxyguanosine & 288.0766 & 244.36 & HILIC (-) & 1 \\
\hline Betaine & 118.0875 & 252.35 & HILIC (+) & 1 & Methylguanosine & 296.1032 & 175.93 & HILIC (-) & 1 \\
\hline Taurine & 124.0067 & 274.34 & HILIC (-) & 1 & Sphingosine & 300.2892 & 345.32 & RPLC (+) & 1 \\
\hline Leucine & 130.0870 & 254.11 & HILIC (-) & 1 & Linoleic acid & 301.2151 & 446.65 & RPLC (-) & 1 \\
\hline Aspartate & 132.0296 & 394.38 & HILIC (-) & 1 & Arachidonic acid & 303.2329 & 438.21 & RPLC (-) & 1 \\
\hline Malate & 133.0136 & 392.16 & HILIC (-) & 1 & Dimethylguanosine & 310.1149 & 177.18 & HILIC (-) & 1 \\
\hline Homocysteine & 134.0281 & 276.82 & HILIC (-) & 1 & Palmitoylcarnitine & 400.3411 & 451.71 & RPLC (+) & 1 \\
\hline Hypoxanthine & 135.0313 & 155.80 & HILIC (-) & 1 & LPA (18:2) & 433.2346 & 391.19 & RPLC (-) & 1 \\
\hline Glutamate & 146.0443 & 384.25 & HILIC (-) & 1 & $\operatorname{LPE}(16: 1)$ & 450.2629 & 440.03 & RPLC (-) & 1 \\
\hline Glutamine & 147.0761 & 351.55 & HILIC (+) & 1 & LPA $(20: 4)$ & 457.2336 & 390.28 & RPLC (-) & 1 \\
\hline Methionine & 150.0582 & 262.92 & HILIC (+) & 1 & LPA (20:2) & 461.2640 & 421.74 & RPLC (-) & 1 \\
\hline Creatine & 152.0431 & 324.68 & HILIC (-) & 1 & LPE (P18:0) & 464.3127 & 469.59 & RPLC (-) & 1 \\
\hline 2,3-Dihydroxybenzoic acid & 153.0191 & 24.34 & HILIC (-) & 1 & $\operatorname{LPC}(14: 1)$ & 466.2936 & 338.73 & RPLC (+) & 1 \\
\hline Carnitine & 162.1138 & 329.69 & HILIC (+) & 1 & $\operatorname{LPC}(14: 0)$ & 468.3080 & 366.14 & RPLC (+) & 1 \\
\hline Phenylalanine & 164.0712 & 243.41 & HILIC (-) & 1 & $\operatorname{LPE}(16: 0)$ & 476.2734 & 410.72 & RPLC (+) & 1 \\
\hline 3-Methylxanthine & 165.0422 & 117.62 & HILIC (-) & 1 & LPE (18:2) & 478.2940 & 390.22 & RPLC (+) & 1 \\
\hline Acetyl-aspartic acid & 174.0392 & 385.51 & HILIC (-) & 1 & $\operatorname{LPE}(18: 1)$ & 480.3083 & 420.86 & RPLC (+) & 1 \\
\hline Citrulline & 174.0878 & 368.82 & HILIC (-) & 1 & LPE (18:0) & 482.3233 & 459.26 & RPLC (+) & 1 \\
\hline Arginine & 175.1193 & 481.99 & HILIC (+) & 1 & LPA (22:5) & 483.2481 & 390.83 & RPLC (-) & 1 \\
\hline Formylmethionine & 176.0382 & 181.34 & HILIC (-) & 1 & LPE (20:3) & 502.2914 & 405.06 & RPLC (-) & 1 \\
\hline Hydroxyphenyllactic acid & 181.0499 & 175.93 & HILIC (-) & 1 & LPE (20:4) & 502.2923 & 389.41 & RPLC (+) & 1 \\
\hline Tyrosine & 182.0801 & 279.34 & HILIC (+) & 1 & LPC $(16: 1)$ & 516.3054 & 377.56 & RPLC (+) & 1 \\
\hline Kynurenic acid & 188.0341 & 175.14 & HILIC (-) & 1 & $\operatorname{LPC}(18: 3)$ & 518.3234 & 368.19 & RPLC (+) & 1 \\
\hline Indole-3-lactic acid & 188.0699 & 158.15 & RPLC (+) & 1 & LPC (18:0) & 524.3717 & 451.71 & RPLC (+) & 1 \\
\hline Kynurenine & 190.0497 & 242.16 & HILIC (-) & 1 & LPE (22:6) & 526.2919 & 387.06 & RPLC (+) & 1 \\
\hline Glucuronic acid & 193.0352 & 375.13 & HILIC (-) & 1 & GCDCA-7-sulfate & 528.2630 & 225.13 & HILIC (-) & 1 \\
\hline Cysteine-S-sulfate & 199.9688 & 287.69 & HILIC (-) & 1 & LPC (20:4) & 544.3391 & 391.22 & RPLC (+) & 1 \\
\hline Hydroxykynurenamine & 203.0815 & 222.28 & HILIC (+) & 1 & LPC (20:3) & 546.3547 & 460.98 & RPLC (+) & 1 \\
\hline Acetylcarnitine & 204.1230 & 284.99 & HILIC (+) & 1 & LPC (22:6) & 568.3403 & 388.33 & RPLC (+) & 1 \\
\hline Tryptophan & 205.0965 & 239.27 & HILIC (+) & 1 & $\operatorname{LPC}(22: 5)$ & 592.3366 & 399.69 & RPLC (+) & 1 \\
\hline Myristic acid & 227.2002 & 415.44 & RPLC (-) & 1 & PE (38:6) & 762.5074 & 512.62 & RPLC (-) & 1 \\
\hline Pseudouridine & 243.0622 & 224.53 & HILIC (-) & 1 & & & & & \\
\hline
\end{tabular}

$\mathrm{m} / z$; mass to charge ratio, RT; retention time, MSI; metabolomics standards initiative, HILIC; hydrophilic interaction liquid chromatography, RPLC; reversed phase liquid chromatography, LPA; lysophosphatidic acid, LPE; lysophosphatidylethanolamine, LPC; lysophosphatidylcholine, GCDCA-7-sulfate; 
medRxiv preprint doi: https://doi.org/10.1101/2020.09.06.20189159; this version posted September 8, 2020. The copyright holder for this preprint

(which was not certified by peer review) is the author/funder, who has granted medRxiv a license to display the preprint in perpetuity.

It is made available under a CC-BY-NC-ND 4.0 International license .

glycochenodeoxycholate 7-suleate, PE; phosphatidylcholine.* Liquid chromatography-mass spectrometry analysis mode, detailing chromatography type (HILIC or RPLC) and electrospray ionization mode (+) or (-). MSI: metabolomics standards initiative level of metabolite identification ${ }^{41}$. 
Extended Data Table 3. Multivariable logistic regression analysis of metabolite levels from healthcare workers and COVID-19 patients.

\begin{tabular}{lccl}
\hline Metabolite & $\beta$ & $p$ value & $q$ value \\
\hline Glutamate & 6.43 & 0.0018 & 0.0228 \\
Glutamine & -12.1 & 0.0014 & 0.0228 \\
Cysteine-S-sulfate & 3.67 & 0.0014 & 0.0228 \\
3-Methylxanthine & -2.10 & 0.0015 & 0.0228 \\
Palmitoleic acid & 4.72 & 0.0008 & 0.0228 \\
Arachidonic acid & 6.35 & 0.0015 & 0.0228 \\
Tryptophan & -5.04 & 0.0044 & 0.0296 \\
Proline & -5.77 & 0.0049 & 0.0296 \\
Citrulline & -3.82 & 0.0051 & 0.0296 \\
Homoserine & -6.43 & 0.0041 & 0.0296 \\
2,3-Dihydroxybenzoic acid & -3.11 & 0.0032 & 0.0296 \\
LPA (18:2) & -4.79 & 0.0050 & 0.0296 \\
LPE (22:6) & 4.74 & 0.0040 & 0.0296 \\
LPA (20:2) & -4.40 & 0.0058 & 0.0313 \\
Uracil & 5.04 & 0.0095 & 0.0467 \\
Myristic acid & 3.88 & 0.0100 & 0.0467 \\
LPC (14:0) & -7.23 & 0.0106 & 0.0467 \\
\hline
\end{tabular}

Models were adjusted by age, sex, and BMI. $q$-values Benjamini-Hochberg adjusted $p$ value. LPA; lysophosphatidic acid, LPE; lysophosphatidylethanolamine, LPC; lysophosphatidylcholine 